\title{
Avifauna (Passeriformes) of Santa Cruz province, Patagonia (Argentina): annotated list of species
}

\author{
Carlos Aquiles DARRIEU ${ }^{1}$, Aníbal Raúl CAMPERI ${ }^{1,2} \&$ Santiago IMBERTI ${ }^{3}$
}

\begin{abstract}
${ }^{1}$ División Zoología Vertebrados, Sección Ornitología, Museo de La Plata, Paseo del Bosque, 1900 La Plata, Pcia. de Buenos Aires, cdarrieu@fcnym.unlp.edu.ar. ${ }^{2}$ Adscripto a la División Ornitología, Museo Argentino de Ciencias Naturales "Bernardino Rivadavia", Av. A. Gallardo 470, C1405DJR Buenos Aires, acamperi@fcnym.unlp.edu.ar. ${ }^{3}$ Rivadavia 780,9400 Río Gallegos, Santa Cruz, imbertis@ar.inter.net
\end{abstract}

\begin{abstract}
The birds (Passeriformes) of Santa Cruz province, Argentina, are analyzed based on three main sources: specimens housed in the Museo Argentino de Ciencias Naturales of Buenos Aires (MACN), in the Félix de Azara Collection, Buenos Aires (CFA), in the Museo de La Plata, La Plata (MLP), in the Fundación Miguel Lillo, Tucumán (FML) and in the National Museum of Natural History, Washington (USNM). The data were obtained from bibliographical citations which include precise localities and from field observations. A list of 75 species belonging to 13 families is included. First records with precise localities are provided for five species. New localities are cited for 64 species.
\end{abstract}

Key words: Birds, systematic list, distribution, Santa Cruz, Argentina.

Resumen: Avifauna (Passeriformes) de la provincia de Santa Cruz, Patagonia (Argentina): lista comentada de especies. En el presente trabajo se aporta una lista comentada de todas las especies de aves Passeriformes registradas en la provincia de Santa Cruz. Los ejemplares de colección pertenecen al Museo Argentino de Ciencias Naturales y a la Colección Félix de Azara, ambos de Buenos Aires, al Museo de La Plata, a la Fundación Miguel Lillo de Tucumán y al National Museum of Natural History, Washington. Los datos fueron obtenidos de la literatura, del análisis de especimenes de museos y de observaciones de campo. Esto nos permitió incluir un total de 75 especies pertenecientes a 13 familias. Cinco de ellas no presentaban registros concretos para la provincia, aportándose nuevas localidades para otras 64 .

Palabras clave: Aves, lista sistemática, distribución, Santa Cruz, Argentina.

\section{INTRODUCTION}

This paper completes a study of the Santa Cruz province avifauna, initiated with the non Passeriformes analysis, where physiographical data are provided (Darrieu et al., 2008).

Although there are an important number of papers relating to different aspects of the ornithological fauna of Santa Cruz, all of them refer to particular areas or singular species that have been studied from a systematic or biological point of view. Among them it is possible to mention Zapata (1967, 1969), Daciuk (1977), De Lucca \& Saggese (1992), Albrieu et al. (2004) and Imberti (2003, 2005). This contribution is the first list that makes a complete revision of the occurrence of each species in Santa Cruz province. It was compiled according to bibliographic data, skins from different museum collections and field observations.

A total of 75 species belonging to 13 families of Passeriformes were registred. New localities for 64 species are derived from collected mate- rial and field observations. 174 localities (5 not localized) are mentioned and 35 pin-pointed on the map (Fig. 1) (See Materials and Methods).

\section{MATERIALS AND METHODS}

A revision of the ornithological collection from the province of Santa Cruz, stored in the Museo Argentino de Ciencias Naturales, Buenos Aires (MACN), the Félix de Azara Collection, Buenos Aires (CFA), the Museo de La Plata, Buenos Aires (MLP), the Fundación Miguel Lillo, Tucumán (FML) and the National Museum of Natural History, Washington (USNM) is provided in the present work

The taxonomy follows Del Hoyo et al. (2003, 2004, 2005, 2006) and Ridgely \& Tudor (1994) and the systematic sequence follows Mazar Barnett \& Pearman (2001). Locality, number of individuals, sex (M: male; F: female), date of capture, collector and place of storage are given for the specimens in the collections mentioned above. 
Species not found in the collections but cited by other authors are included as well as observations made by the authors and other specialists (C. Albrieu, M. Díaz, A. Earnshaw, A. Falcone, S. Ferrari, M. McNamara, E. Militello, P. and S. Sturzenbaum, L. Bernacci, M. Bertinat, J. Schieda, V. Sotelo, J. Zancaner and C. Zoratti). Subspecific assignment was made according to geographic distribution in the cases where specimens were not available.

Localities are ordered alphabetically with a number assigned for each location on the map in Appendix 1. The catalogue of Paynter Jr. (1995) and a Geographic Atlas of the Argentine Republic edited by the Centro Cartográfico Buenos Aires S.R.L were used to locate each collecting locality. Localities are numbered from north to south on the map (Fig. 1). Various new localities, not previously mapped for the non Passerines (see Darrieu et al., 2008), are included in the present map.

\section{ANNOTATED CHECKLIST}

\section{FURNARIIDAE}

\section{Geositta cunicularia cunicularia}

Literature. Río Gallegos (Dabbene, 1919); Paso Ibáñez, Río Gallegos, Río Coig (Esteban, 1951); Puerto Deseado, Puerto San Julián (Humphrey et al., 1985); La Esperanza (de la Peña, 1987); Estancia El Cuadro (De Lucca \& Saggese, 1992); Monumento Natural Bosques Petrificados, Parque Nacional Los Glaciares, Parque Nacional Perito Moreno (Chebez et al., 1998); Laguna Nimez (Imberti \& Albrieu, 2001); Parque Nacional Los Glaciares (Sección Centro), Lago Roca, Glaciar Perito Moreno, El Chaltén, Bahía del Túnel (Imberti, 2005); Estancia El Cóndor (Coconier, 2005); Río Gallegos, Río Coig, Meseta Lago Strobel, Río Chico, Monumento Natural Bosques Petrificados, Estancia El Cuadro, Parque Nacional Perito Moreno, Meseta Lago Buenos Aires, El Zurdo, Laguna Nimez, Lago Argentino, Meseta del Asador, Parque Nacional Los Glaciares, Estancia La Soledad, Estancia La Anita, Estancia El Cóndor, Bahía San Julián (Di Giácomo et al., 2005).

Museum specimens. Estancia El Tranquilo, 1 M, 29 Jan 1971; Estancia Roca Blanca, 4 M, 2 F and 1 ?, 22 Dec 1960, 3-16 Jan 1961, 2-29 Jan 1971, col. A. Budin \& J. Viera, FML; Pampa del Asador, 1 ?, 30 Feb 1936, collector unknown, MLP. Field observations. Parque Nacional Perito Moreno, Parque Nacional Los Glaciares, Monumento Natural Bosques Petrificados, Laguna Los Escarchados, Estancia Rincón de los
Morros, Estancia La Angostura, Estancia Rupai Pacha, Cabo Vírgenes, Estancia Pali Aike, Estancia Sofía, Estancia Buitreras, Estancia Bella Vista Bitsch, Estancia La Carlota, Ría Coig, El Zurdo, Estancia El Cóndor, El Roda, Monte León, Koluel Kayke (Imberti).

\section{Geositta antarctica}

Literature. Río Gallegos (Esteban, 1951); Meseta La Siberia, Estancia Rincón de los Toros -Meseta del Lago Cardiel Chico- (Chebez et al., 1988); Río Guanaco (Imberti, 2005); Meseta Lago Strobel, Meseta Lago Buenos Aires, Meseta del Asador, Parque Nacional Los Glaciares, Estancia El Cóndor (Coconier, 2005); Monte León, Río Gallegos, Río Coig, Meseta Lago Strobel, Río Chico, Monumento Natural Bosques Petrificados, Estancia El Cuadro, Parque Nacional Perito Moreno, Meseta Lago Buenos Aires, El Zurdo, Meseta del Asador, Parque Nacional Los Glaciares, Estancia La Soledad, Estancia La Anita, Estancia El Condor, Bahía San Julián (Di Giácomo et al., 2005); Cabo Vírgenes (Imberti, unpubl. a).

Field observations. Estancia La Angostura, Laguna Los Escarchados, Punta Loyola, Tres Cerros, Monte León (Imberti).

\section{Geositta rufipennis giaii}

Literature. Parque Nacional Perito Moreno (Chebez et al., 1998); Río de las Vueltas (Imberti, 2005); Parque Nacional Perito Moreno, Parque Nacional Los Glaciades, Estancia La Soledad, Estancia La Anita (Di Giácomo et al., 2005).

Field observations. Río Pinturas, Río Turbio (southernmost record for the species, Imberti).

\section{Upucerthia dumetaria dumetaria}

Literature. Puerto Deseado (Gould \& Darwin, 1838-1841; Zapata, 1967); Río Deseado (70으르. W) (Burmeister, 1890); Cañadón Misioneros (Oustalet, 1891); Río Chico, Ría Coig (Stone, 1928); Río Gallegos, Río Coig (Esteban, 1951); Punta Bandera (Navas, 1971); Laguna Los Escarchados (Lange, 1981); Río Belgrano (Narosky et al., 1983); Puerto Deseado, Puerto Santa Cruz (Humphrey et al., 1985); Estancia El Cuadro (De Lucca \& Saggese, 1992); Monumento Natural Bosques Petrificados, Parque Nacional Los Glaciares, Parque Nacional Perito Moreno (Chebez et al., 1998); Laguna Nimez (Imberti \& Albrieu 2001); Lago Roca, Bahía del Túnel (Imbertis, 2005); Estancia El Cóndor (Coconier, 2005); Río Gallegos, Río Coig, Meseta Lago Strobel, Río Chico, Monumento Natural Bosques Petrificados, Estancia El Cuadro, Parque Nacional 


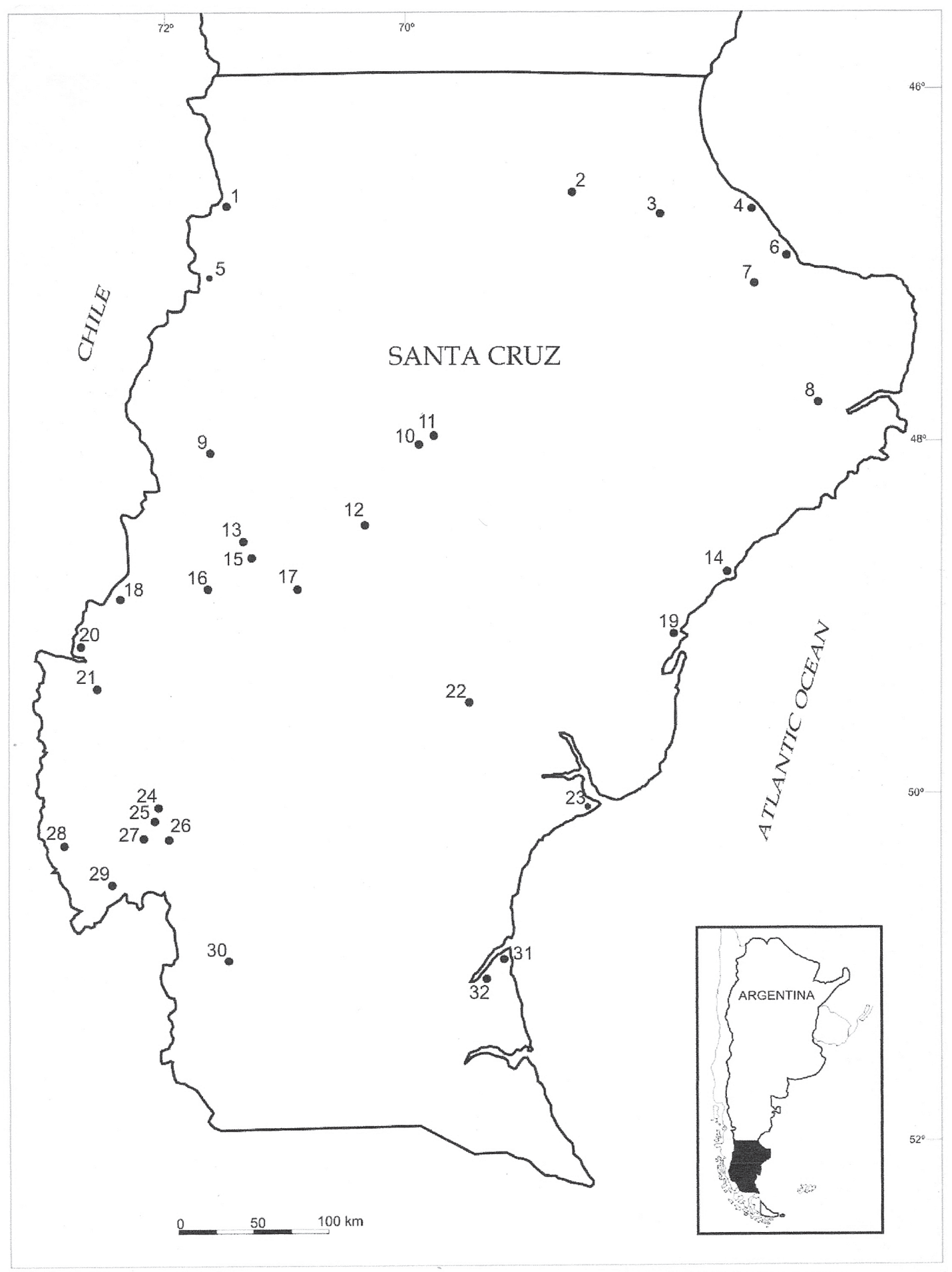

Fig. 1. Localities that had not been cited in the paper about non Passeriformes (Darrieu et al. 2008) 
Perito Moreno, Meseta Lago Buenos Aires, El Zurdo, Laguna Nimez, Lago Argentino, Parque Nacional Los Glaciares, Estancia La Soledad, Estancia La Anita, Estancia El Cóndor, Bahía San Julián (Di Giácomo et al., 2005).

Museum specimens. Estancia El Puma, La Manchuria, 1 ?, 20 Jan 2000, col. Y. Davies, CFA; Lago Argentino, Punta Bandera, 1 F ?, 21 Feb 1959, col. P. Miles; Río Gallegos, 1 F, 29 Nov 1981, col. D.H. Ellis, MACN.

Field observations. Parque Nacional Perito Moreno, Parque Nacional Los Glaciares, Monumento Natural Bosques Petrificados, Estancia Rupai Pacha, Estancia Rincón de los Morros, Río Gallegos, Punta Loyola, Cabo Vírgenes, Laguna Nimez, Río Bote, Ría Coig, Estancia La Angostura, Estancia Bella Vista, Monte León, Koluel Kayke (Imberti).

\section{Cinclodes fuscus fuscus}

Literature. Caprek-aik, Gol-aik (Burmeister, 1890); Río Gallegos (Oustalet, 1891); Puerto Deseado, Río Gallegos (Dabbene, 1919); Puerto Deseado (Zapata, 1967; Daciuk, 1977, Estancia El Cuadro (De Lucca \& Saggese, 1992); Puerto Deseado, Isla Pingüino (Humphrey et al., 1985); Monumento Natural Bosques Petrificados, Parque Nacional Los Glaciares, Parque Nacional Perito Moreno (Chebez et al., 1998); Laguna Nimez (Imberti \& Albrieu, 2001); Parque Nacional Los Glaciares (Sección Centro) (Imberti, 2005); Río Gallegos, Río Coig, Meseta Lago Strobel, Río Chico, Monumento Natural Bosques Petrificados, Estancia El Cuadro, Parque Nacional Perito Moreno, Meseta Lago Buenos Aires, El Zurdo, Laguna Nimez, Lago Argentino, Meseta del Asador, Parque Nacional Los Glaciares, Estancia La Soledad, Estancia La Anita (Di Giácomo et al., 2005).

Museum specimens. Lago Argentino, Lago Frío, 1 M, 18 Jan 1959; Lago Argentino, Ventisquero Onelli, 1 M, 2 Jan 1959, col. P. Miles; Puerto Deseado, 1 M, 10 Aug 1961, col. A. Zapata, MACN. Field observations. Parque Nacional Perito Moreno, Parque Nacional Los Glaciares, Monumento Natural Bosques Petrificados, Estancia Rupai Pacha, Estancia Rincón de los Morros, Río Gallegos, Cabo Vírgenes, Laguna Nimez, Ría Coig, Estancia La Angostura, Bahía Redonda, Estancia 9 de Julio, Estancia Pali Aike, El Zurdo, Estancia Sofía, Laguna Azul, Estancia Bella Vista Bitsch, El Roda, Estancia Bella Vista, Monte León (Imberti).

\section{Cinclodes oustaleti hornensis}

Literature. Monumento Natural Bosques Petrificados, Parque Nacional Los Glaciares, Parque
Nacional Perito Moreno (Chebez et al., 1998); Parque Nacional Los Glaciares, Puesto La Nana (Imberti, 2003); Glaciar Perito Moreno (Imberti, 2005); Parque Nacional Perito Moreno, Parque Nacional Los Glaciares, Estancia La Soledad, Estancia La Anita (Di Giácomo et al., 2005).

Field observations. Parque Nacional Los Glaciares, Estancia Rincón de los Morros, Estancia Cóndor (Imberti).

\section{Cinclodes patagonicus chilensis}

Literature. Río Lista (Narosky et al., 1983); Parque Nacional Los Glaciares, Parque Nacional Perito Moreno (Chebez et al., 1998).

\section{Cinclodes patagonicus patagonicus}

Literature. Laguna Nimez (Imberti \& Albrieu, 2001); Parque Nacional Los Glaciares (Imberti, 2005).

Museum specimens. Lago Argentino, Lago Frío, 1 M ?, 18 Jan 1959, col. P. Miles, MACN.

Field observations. Parque Nacional Los Glaciares, Estancia Rincón de los Morros, Parque Nacional Perito Moreno, Lago Argentino, Estancia Rupai Pacha, Puerto Bandera, Estancia La Soledad, Lago del Desierto, Estancia Glencross, Estancia Bella Vista, Estancia Cóndor (Imberti).

\section{Sylviorthorhynchus desmursii}

Literature. Lago San Martín (Radboone 1935); Parque Nacional Los Glaciares (Chebez et al., 1998); Estancia Cóndor -Puesto La Nana(Imberti, 2003)

Field observations. Estancia Cóndor (Imberti); El Chaltén (Sotelo pers. comm.).

\section{Aphrastura spinicauda spinicauda}

Literature. Parque Nacional Los Glaciares, Parque Nacional Perito Moreno (Chebez et al., 1998); Parque Nacional Los Glaciares (Imberti, 2005); Parque Nacional Perito Moreno, Parque Nacional Los Glaciares, Estancia La Soledad, Estancia La Anita (Di Giácomo et al., 2005).

Museum specimens. Lago Argentino, Cerro Mayo, 1 M, 10 Feb 1959, col. P. Miles, MACN

Field observations. Parque Nacional Los Glaciares, Estancia Rincón de los Morros, Parque Nacional Perito Moreno, Puerto Bandera, Estancia La Soledad, Lago del Desierto, Estancia Glencross, Estancia Morro Chico, Estancia Cóndor (Imberti); Río Gallegos (Sturzenbaum pers. comm.).

\section{Leptasthenura platensis}

Literature. Paso Ibáñez (Humphrey \& Bridge, 1970). 


\section{Leptasthenura aegithaloides pallida}

Literature. Río Gallegos (Stone, 1928); Estancia El Cóndor, Lago Roca (Navas \& Bó, 1987); Cabo Vírgenes, Punta Quilla, Punta Cascajo, Cañadón Leones, Estancia La Unión, Estancia El Cóndor, Lago Argentino, Lago Roca, Lago San Martín (Chebez et al., 1988); Estancia El Cuadro (De Lucca \& Saggese, 1992); Monumento Natural Bosques Petrificados, Parque Nacional Los Glaciares (Chebez et al., 1998); Laguna Nimez (Imberti \& Albrieu, 2001); Lago Roca, El Chaltén, Lago Argentino (Imberti, 2005).

Museum specimens. Lago Argentino, Lago Roca, 1 F, 26 Jan 1959, col. P. Miles; Río Gallegos, 1 M, 28 Apr 1974, col. M. Rumboll, MACN.

Field observations. Parque Nacional Los Glaciares, Monumento Natural Bosques Petrificados, Estancia Rupai Pacha, Ría Coig, Lago Posadas, Cabo Vírgenes, Estancia La Angostura, Laguna Nimez, Estancia La Lucha, Monte León, Koluel Kayke (Imberti).

\section{Asthenes pyrrholeuca pyrrholeuca}

Literature. Puerto Deseado (Sclater, 1874; Hellmayr, 1925); Monte León, Puerto Coig, Punta Quilla (Chebez et al., 1988); Estancia El Cuadro (De Lucca \& Saggese, 1992); Monumento Natural Bosques Petrificados, Parque Nacional Los Glaciares, Parque Nacional Perito Moreno (Chebez et al., 1998); Lago Roca (Imberti, 2005); Río Gallegos, Río Coig, Meseta Lago Strobel, Río Chico, Monumento Natural Bosques Petrificados, Estancia El Cuadro, Meseta Lago Buenos Aires, Laguna Nimez, Lago Argentino, Meseta del Asador, Parque Nacional Los Glaciares, Estancia La Soledad, Estancia La Anita, Estancia El Cóndor (Di Giácomo et al., 2005).

Museum specimens. Bahía del Fondo, $1 \mathrm{M}$ and 1 F, 4 Jan 1922, col. A. Merkle, MLP; Estancia El Puma, La Manchuria, 2 ?, 16 Jan 2000, CFA.

Field observations. Parque Nacional Los Glaciares, Monumento Natural Bosques Petrificados, Estancia La Angostura, Parque Nacional Perito Moreno, Estancia Las Horquetas, Monte León, Koluel Kayke (Imberti).

\section{Asthenes modesta australis}

Literature. Aguada Grande, Río Coig (Contreras, 1980; Navas \& Bó, 1987); Estancia El Cuadro (De Lucca \& Saggese, 1992); Monumento Natural Bosques Petrificados, Parque Nacional Perito Moreno (Chebez et al., 1998); Laguna Nimez (Imberti \& Albrieu, 2001); Bahía del Túnel, Lago Roca (Imberti, 2005); Meseta Lago Strobel, Río Chico, Monumento Natural Bosques Petrificados, Estancia El Cuadro, Parque Nacional Perito Moreno, Meseta Lago Buenos Aires,
Meseta del Asador, Parque Nacional Los Glaciares, Estancia La Soledad, Estancia La Anita (Di Giácomo et al., 2005); Cabo Vírgenes (Imberti unpubl. a).

Museum specimens. Río Coig, ruta 3, 1 M, 3 May 1974, col. M. Rumboll, MACN.

Field observations. Monumento Natural Bosques Petrificados, Parque Nacional Perito Moreno, Punta Loyola, Estancia El Cóndor, Ría Coig, Estancia La Angostura, Estancia Buitreras, Monte León (Imberti).

\section{Asthenes patagonica}

Literature. Monumento Natural Bosques Petrificados (Ridgely \& Tudor, 1994; Chebez et al., 1998); Monumento Natural Bosques Petrificados, Estancia El Cuadro (Di Giácomo et al., 2005).

Field observations. East of Gobernador Gregores, Puerto Deseado (Imberti).

\section{Asthenes anthoides}

Literature. Río Ecker (Stone, 1928); Parque Nacional Los Glaciares (Chebez et al., 1998); Laguna Nimez (Imberti \& Albrieu, 2001); Río Bote, El Calafate, Estancia La Soledad, Estancia Cristina, from Río Gallegos to Cabo Vírgenes (Imberti, 2003); Puerto Bandera, Estancia Cristina, Estancia Helsingfors, Lago Roca (Imberti, 2005); Río Gallegos, Parque Nacional Los Glaciares, Estancia El Cóndor (Coconier, 2005); Río Gallegos, Parque Nacional Perito Moreno, Laguna Nimez, Lago Argentino, Parque Nacional Los Glaciares, Estancia La Soledad, Estancia La Anita, Estancia El Cóndor (Di Giácomo et al., 2005).

Museum specimens. Río Gallegos, 1 M, 28 Apr 1974, 1 F ?, 1 May 1974, col. M. Rumboll, MACN. Field observations. Parque Nacional Perito Moreno, Parque Nacional Los Glaciares, Punta Loyola, Estancia El Cóndor, Bahía Redonda, Laguna Nimez, Cabo Vírgenes, Puerto Bandera, Estancia La Soledad, Monte León (Imberti).

\section{Phleocryptes melanops melanops}

Literature. Río Chico (Stone, 1928; Coconier, 2005); Estancia Alta Vista, Punta Bandera, Perito Moreno (Chebez et al., 1988); Estancia La Angostura, Río Chico (Mazar Barnett et al.,1998); Laguna Nimez (Imberti \& Albrieu, 2001); El Zurdo, Laguna Nimez, Estancia La Angostura, Estancia San Lorenzo, Estancia 9 de Julio, Estancia El Cóndor, Estancia La Alice, Estancia Coy Aike, Estancia La Soledad, Estancia María Aike (Imberti, 2003); Bahía del Túnel, Estancia San José, Puerto Bandera, Estancia Cristina, Estancia Anita (Imberti, 2005). 
Field observations. El Zurdo, Laguna Nimez, Estancia La Angostura, Estancia San Lorenzo, Estancia 9 de Julio, Estancia Cóndor, Estancia La Alice, Estancia Coy Aike, Estancia María Aike (Imberti).

\section{Eremobius phoenicurus}

Literature. Puerto Deseado, San Julián, Santa Cruz (Gould \& Darwin, 1838-1841; Sclater, 1890; Stone, 1928); Río Ecker (Scott, 1900b); Río Gallegos (Olrog, 1948); Paso Ibáñez (Esteban, 1951); Puerto Deseado (Zapata, 1967, Humphrey et al., 1985); Bahía del Fondo, Puerto Deseado (Navas, 1971); Estancia El Cuadro, Monumento Natural Bosques Petrificados (De Lucca \& Saggese, 1992); Monumento Natural Bosques Petrificados (Chebez et al., 1998); Laguna Nimez (Imberti \& Albrieu, 2001); Río Coig (Coconier, 2005); Río Coig, Monumento Natural Bosques Petrificados, Estancia El Cuadro, Bahía San Julián (Di Giácomo et al., 2005); Estancia La Angostura (Imberti unpubl. b).

Museum specimens. Bahía del Fondo, $1 \mathrm{M}, 4$ Jan 1922, col. A. Merkle, MLP; Caleta Olivia, 1 M, 6 May 1974, col. M. Rumboll; Jaramillo, 1 M, 23 Feb 1981, col. M. I. Christie; Puerto Deseado, 1 M, 17 Aug 1961, col. A. Zapata; Río Coig, ruta 3, $1 \mathrm{M}$ and $1 \mathrm{~F}, 3$ May 1974, col. M. Rumboll, MACN.

Field observations. Monumento Natural Bosques Petrificados, Estancia Rupai Pacha, Ría Coig, Estancia Coy Aike, Monte León, Koluel Kayke, Estancia Punta Loyola, Estancia Coy Inlet (Imberti).

\section{Pseudoseisura gutturalis gutturalis}

Literature. Puerto Deseado (Zapata, 1967; Daciuk, 1977); Fitz Roy, Mazarredo (Zapata, 1969); Cañadón Leones (Chebez et al., 1988); Monumento Natural Bosques Petrificados (Chebez et al., 1998); El Chaltén (Imberti, 2005); Río Chico, Monumento Natural Bosques Petrificados, Estancia El Cuadro, Parque Nacional Los Glaciares, Estancia La Soledad, Estancia La Anita, Bahía San Julián (Di Giácomo et al., 2005).

Museum specimens. Bahía del Fondo, 2 M, 14 Dec 1921, 4 Jan 1922, col. A. Merkle, MLP.

Field observations. Monumento Natural Bosques Petrificados, Estancia La Angostura, Estancia La Lucha, Monte León, Koluel Kayke (Imberti).

\section{Pygarrhichas albogularis}

Literature. Lago San Martín (Radboone, 1935); Parque Nacional Los Glaciares, Parque Nacional Perito Moreno (Chebez et al., 1998); Parque
Nacional Los Glaciares (Imberti,2005); Parque Nacional Perito Moreno, Parque Nacional Los Glaciares, Estancia La Soledad, Estancia La Anita (Di Giácomo et al., 2005).

Museum specimens. Lago Argentino, Cerro Mayo, $1 \mathrm{M}$ and $1 \mathrm{~F}, 5$ and $14 \mathrm{Feb}$ 1959, col. P. Miles, MACN.

Field observations. Parque Nacional Los Glaciares, Parque Nacional Perito Moreno, Estancia Cóndor, Estancia Tucu Tucu (Imberti).

\section{RHINOCRYPTIDAE}

\section{Pteroptochos tarnii}

Literature. Parque Nacional Los Glaciares, Parque Nacional Perito Moreno (Chebez et al., 1998), Río Blanco, El Chaltén, Puesto La Nana, Parque Nacional Perito Moreno (Imberti, 2003); El Chaltén, Río Blanco, Glaciar Perito Moreno (Imberti, 2005); Parque Nacional Perito Moreno, Parque Nacional Los Glaciares, Estancia La Soledad, Estancia La Anita (Di Giácomo et al., 2005).

Field observations. Parque Nacional Perito Moreno, Parque Nacional Los Glaciares, Estancia Cóndor (Imberti).

\section{Scytalopus magellanicus}

Literature. Parque Nacional Los Glaciares, Parque Nacional Perito Moreno (Chebez et al., 1998); Parque Nacional Los Glaciares (Imberti, 2005); Parque Nacional Perito Moreno, Parque Nacional Los Glaciares, Estancia La Soledad, Estancia La Anita (Di Giácomo et al., 2005).

Museum specimens. Lago Argentino, Cerro Mayo, $1 \mathrm{M}$ and 1 F, 9 Feb 1959, col. P. Miles, MACN.

Field observations. Parque Nacional Perito Moreno, Parque Nacional Los Glaciares, Lago del Desierto, Estancia Tucu Tucu (Imberti).

\section{TYRANNIDAE}

\section{Elaenia albiceps chilensis}

Literature. Cabo Dañoso, Lago Strobel, Estancia Las Tunas, Estancia Rincón de los Toros (Chebez et al., 1998); Parque Nacional Los Glaciares, Parque Nacional Perito Moreno (Chebez et al., 1998); Los Antiguos (Earnshaw \& Earnshaw, 2000); Parque Nacional Los Glaciares (Imberti, 2005); Parque Nacional Perito Moreno, Laguna Nimez, Lago Argentino, Parque Nacional Los Glaciares, Estancia La Soledad, Estancia La Anita (Di Giácomo et al., 2005).

Museum specimens. Lago Argentino, Lago Roca, 1 M, 26 Jan 1959, col. P. Miles, MACN. 
Field observations. Parque Nacional Perito Moreno, Parque Nacional Los Glaciares, Lago Posadas, Lago del Desierto, Estancia Rincón de los Morros, Estancia Rupai Pacha, Estancia Las Tunas, Estancia Glencross, Estancia La Angostura, Estancia La Quinta, El Pluma, Estancia María Cristina (Imberti); Puerto Deseado (Bertinat pers. comm.).

\section{Anairetes parulus parulus}

Literature. Lago San Martín, Lago Argentino (Navas \& Bó, 1991); Monumento Natural Bosques Petrificados, Parque Nacional Los Glaciares, Parque Nacional Perito Moreno (Chebez et al., 1998); Laguna Nimez (Imberti \& Albrieu, 2001); Parque Nacional Los Glaciares (Imberti, 2005).

Museum specimens. Lago Argentino, brazo Sud, $1 \mathrm{M}$ and 1 F, 14 Jan 1959, col. P. Miles; Lago San Martín, 1 M and 1 ?, Dec 1936, Feb 1939, MACN. Field observations. Parque Nacional Perito Moreno, Parque Nacional Los Glaciares, Lago Posadas, Estancia Rincón de los Morros, Estancia La Angostura, Estancia María Cristina, Río Turbio, Parque Nacional Monte León (Imberti). Anairetes parulus ssp.

Literature. Estancia El Cuadro (De Lucca \& Saggese, 1992).

Field observations. Monumento Natural Bosques Petrificados, Estancia Cóndor (Imberti).

\section{Tachuris rubrigastra rubrigastra}

Literature. Estancia La Angostura, Río Chico (Mazar Barnett et al., 1998); Puerto Bandera (Iglesias \& Pérez, 1998); Laguna Nimez (Imberti \& Albrieu, 2001); Puerto Bandera, Laguna Nimez, Estancia La Angostura, Estancia La Soledad, Estancia 9 de Julio, Estancia San Lorenzo, Estancia La Alice (Imberti, 2003); Bahía del Túnel, Puerto Bandera, Estancia Anita (Imberti, 2005); Río Chico (Coconier, 2005).

Field observations. Estancia La Angostura, Puerto Bandera, Estancia La Soledad, Laguna Nimez, Estancia 9 de Julio, Estancia San Lorenzo, Estancia La Alice (Imberti).

\section{Colorhamphus parvirostris}

Literature. Lago San Martín (Chesser \& Marín, 1994); Parque Nacional Los Glaciares (Chebez et al., 1998); Glaciar Perito Moreno, Lago Roca, El Chaltén (Imberti, 2005).

Field observations. Parque Nacional Los Glaciares (Imberti).

\section{Xolmis pyrope pyrope}

Literature. Río Chico (Stone, 1928); Lago San Martín (Radboone, 1935; Pergolani de Costa,
1949); Parque Nacional Los Glaciares, Parque Nacional Perito Moreno (Chebez et al., 1998); Parque Nacional Los Glaciares (Imberti, 2005); Estancia La Angostura (Imberti unpubl. b).

Museum specimens. Lago Argentino, Ventisquero Onelli, 1 F, 5 Jan 1959, col. P. Miles, MACN.

Field observations. Parque Nacional Perito Moreno, Parque Nacional Los Glaciares, Estancia Cóndor, Estancia Rincón de los Morros, Estancia Glencross, Río Turbio (Imberti).

\section{Neoxolmis rufiventris}

Literature. Río Coig, Estancia Pali Aike (Stone, 1928); Puerto Deseado (Zapata, 1967; de la Peña, 1987, 2005); Mazarredo (Zapata, 1969); Estancia Las Vegas (Maclean 1969); Laguna Los Escarchados (Lange, 1981); Reserva de Vida Silvestre Los Escarchados (Erize, 1983); Puerto Deseado (de la Peña, 1985); Estancia El Cuadro (De Lucca \& Saggese, 1992); Parque Nacional Los Glaciares, Parque Nacional Perito Moreno (Chebez et al., 1998); Parque Nacional Los Glaciares (Sección Centro), Bahía del Túnel, Lago Roca (Imberti, 2005); Meseta Lago Strobel, Río Chico, Meseta Lago Buenos Aires, Meseta del Asador, Estancia El Cóndor (Coconier, 2005); Río Gallegos, Río Coig, Meseta Lago Strobel, Río Chico, Monumento Natural Bosques Petrificados, Estancia El Cuadro, Parque Nacional Perito Moreno, Meseta Lago Buenos Aires, El Zurdo, Laguna Nimez, Lago Argentino, Meseta del Asador, Parque Nacional Los Glaciares, Estancia La Soledad, Estancia La Anita, Estancia El Cóndor, Bahía San Julián (Di Giácomo et al., 2005).

Museum specimens. Cabo Vírgenes, 1 F, 12 Oct 1941, MLP; Puerto Deseado, 1 M, 24 Sep 1961, col. A. Zapata; Río Gallegos, $1 \mathrm{M}$ and 1 F, 29 Nov 1981, col. D.H. Ellis, MACN.

Field observations. Parque Nacional Perito Moreno, Parque Nacional Los Glaciares, Monumento Natural Bosques Petrificados, Estancia El Cóndor, Estancia La Angostura, Cabo Vírgenes, Laguna Los Escarchados, Estancia Rupai Pacha, Punta Loyola, El Roda, Monte León, Koluel Kayke, Estancia María Cristina, Río Santa Cruz, Estancia Luz Divina (Imberti).

\section{Neoxolmis rubetra}

Literature. Estancia El Cuadro (De Lucca \& Saggese, 1992;, De Lucca et al., 1993); Monumento Natural Bosques Petrificados, Estancia El Cuadro (Di Giácomo et al., 2005).

Field observations. Koluel Kayke, Puerto Deseado, Cabo Blanco, Bahía Laura (Imberti). 


\section{Agriornis montana leucurus}

Literature. Río Ecker (Scott, 1900a); Río Ecker, Puerto Deseado (Stone, 1928); Punta Quilla, Punta Bandera, Meseta El Moro, Puesto Ensenada, Lago San Martín, Paso Roballos, Estancia Rincón de los Toros (Chebez et al., 1988); Parque Nacional Los Glaciares, Parque Nacional Perito Moreno (Chebez et al., 1998); Lago Roca, Río de las Vueltas (Imberti, 2005).

Field observations. Parque Nacional Perito Moreno, Monumento Natural Bosques Petrificados, Lago Argentino, lagos del Furioso, Río Pinturas, Estancia La Angostura, Monte León (Imberti).

\section{Agriornis livida fortis}

Literature. Lago San Martín (Pergolani de Costa, 1946); Parque Nacional Los Glaciares, Lago San Martín, El Calafate (Chebez et al., 1988); Punta Bandera (Navas \& Bó, 1994); Parque Nacional Los Glaciares (Chebez et al., 1998); El Chaltén, Lago Roca, Puerto Bandera, Glaciar Perito Moreno, Cerro Torre, El Calafate (Imberti, 2005); Estancia La Angostura (Imberti unpubl. b).

Museum specimens. Lago Argentino, Punta Bandera, 1 M, 22 Feb 1959, col. P. Miles, MACN. Field observations. Parque Nacional Perito Moreno, Parque Nacional Los Glaciares, Estancia Cóndor, Lago Argentino, Estancia La Soledad, Monte León, Koluel Kayke (Imberti).

\section{Agriornis microptera microptera}

Literature. Puerto Deseado, San Julián (Gould \& Darwin, 1838-1841; Sclater, 1888; Zapata \& Martínez, 1972); Puerto Deseado, Cañadón del Puerto, Ría Deseado (Humphrey et al., 1985); Punta Cascajo (Chebez et al., 1988); Estancia El Cuadro (De Lucca \& Saggese, 1992); Punta Avellaneda (Administración Parques Nacionales 1980-90); Monumento Natural Bosques Petrificados, Parque Nacional Los Glaciares (Chebez et al., 1998); Estancia La Angostura (Imberti unpubl. b).

Field observations. Parque Nacional Perito Moreno, Parque Nacional Los Glaciares, Monumento Natural Bosques Petrificados, Bajada de Miguez, Lago Argentino, Monte León, Koluel Kayke (Imberti).

\section{Agriornis murina}

Literature. Cañadón Darwin (Chebez et al., 1988); Monumento Natural Bosques Petrificados, Estancia El Cuadro (Di Giácomo et al., 2005); Estancia La Angostura (Imberti unpubl. b).

Field observations. Koluel Kayke, Puerto San Julián (Imberti).
Muscisaxicola maculirostris maculirostris Literature. San Julián (Gould \& Darwin, 18381841); Caprek-aik (Burmeister, 1890); Estancia El Cuadro, Monumento Natural Bosques Petrificados (De Lucca \& Saggese, 1992); Monumento Natural Bosques Petrificados, Parque Nacional Perito Moreno (Chebez et al., 1998); Río Mitre, Lago Argentino, Bahía del Túnel, Brazo Rico, El Calafate, Lago Argentino (Imberti, 2005); Estancia La Angostura (Imberti unpubl. b). Field observations. Parque Nacional Perito Moreno, Monumento Natural Bosques Petrificado, Estancia Rincón de los Morros, Estancia Palermo Aike, Estancia Rupai Pacha, Cabo Vírgenes, Laguna Los Escarchados, Estancia Pali Aike, Río La Leona, Estancia La Angostura, Estancia Las Tunas (Imberti).

\section{Muscisaxicola macloviana mentalis}

Literature. Río Chico (Stone, 1928); Caleta Olivia, Ventisquero Onelli, Cerro Mayo, Aguada Grande (Navas \& Bó, 1994); Monumento Natural Bosques Petrificados, Parque Nacional Los Glaciares, Parque Nacional Perito Moreno (Chebez et al., 1998); Laguna Nimez (Imberti \& Albrieu, 2001); Parque Nacional Los Glaciares (Imberti, 2005); Monte León, Río Gallegos, Río Coig, Meseta Lago Strobel, Río Chico, Monumento Natural Bosques Petrificados, Estancia El Cuadro, Parque Nacional Perito Moreno, Meseta Lago Buenos Aires, El Zurdo, Laguna Nimez, Lago Argentino, Meseta del Asador, Parque Nacional Los Glaciares, Estancia La Soledad, Estancia La Anita, Estancia El Cóndor, Bahía San Julián (Di Giácomo et al., 2005); Cabo Vírgenes (Imberti unpubl. a); Estancia La Angostura (Imberti unpubl. b).

Museum specimens. Caleta Olivia, $1 \mathrm{M}, 6$ May 1974, col. M. Rumboll; Cerro Mayo, 1 F, 11 Feb 1959; Ventisquero Onelli, 1 F, 5 Jan 1959, col. P. Miles, MACN.

Field observations. Parque Nacional Perito Moreno, Parque Nacional Los Glaciares, Monumento Natural Bosques Petrificados, Lago Posadas, Estancia Rupai Pacha, Punta Loyola, Lago del Desierto, Estancia 9 de Julio, Ría Santa Cruz, Bahía San Julián, El Roda, Monte León (Imberti).

\section{Muscisaxicola albilora}

Literature. Lago Argentino (Navas \& Bó, 1994); Parque Nacional Los Glaciares, Parque Nacional Perito Moreno (Chebez et al., 1998); El Chaltén (Imberti, 2005); Meseta Lago Strobel, Parque Nacional Perito Moreno, Meseta Lago Buenos Aires, Meseta del Asador, Parque Nacional, Estancia La Soledad, Estancia La Anita (Di 
Giácomo et al., 2005); Estancia La Angostura (Imberti unpubl. b).

Museum specimens. Lago Argentino, brazo Sud, 1 F, 12 Jan 1959, col. P. Miles, MACN.

Field observations. Parque Nacional Perito Moreno, Parque Nacional Los Glaciares, Estancia Rupai Pacha, Estancia Bella Vista Bitsch (Imberti).

\section{Muscisaxicola capistrata}

Literature. Ría Coig (Scott 1900a; Stone, 1928); Puerto Deseado (Zapata, 1967); Cabo Vírgenes, Monte León, Cañadón Darwin, Bahía San Julián, Cabo Curioso, Cabo Blanco, Meseta de las Vizcachas, Lago Viedma, Meseta La Siberia, Meseta del Strobel, Meseta del Cardiel Chico, Lago Buenos Aires (Chebez et al., 1988); Estancia El Cuadro, Monumento Natural Bosques Petrificados (De Lucca \& Saggese, 1992); Jaramillo (Navas \& Bó, 1994); Monumento Natural Bosques Petrificados, Parque Nacional Perito Moreno (Chebez et al., 1998); Parque Nacional Los Glaciares (Sección Centro), El Chaltén (Imberti, 2005); Río Gallegos, Río Coig, Meseta Lago Strobel, Río Chico, Monumento Natural Bosques Petrificados, Estancia El Cuadro, Parque Nacional Perito Moreno, Meseta Lago Buenos Aires, Laguna Nimez, Lago Argentino, Meseta del Asador, Parque Nacional Los Glaciares, Estancia La Soledad, Estancia La Anita, Estancia El Cóndor, Bahía San Julián (Di Giácomo et al., 2005); Estancia La Angostura (Imberti unpubl. b). Museum specimens. Lago Chico, sur de Jaramillo, 1 ?, 27 Nov 1981, col. D.H. Ellis; Puerto Deseado, 1 M, 28 Nov 1961, col. A. Zapata, MACN. Field observations. Parque Nacional Perito Moreno, Parque Nacional Los Glaciares, Monumento Natural Bosques Petrificados, Estancia Bella Vista Bitsch, Punta Loyola, Cabo Vírgenes, Laguna Los Escarchados, Estancia Pali Aike, Estancia Cabo Buen Tiempo, Parque Nacional Monte León, Estancia María Cristina (Imberti).

\section{Muscisaxicola flavinucha flavinucha}

Literature. Alto Río Chico (Scott, 1900a); Río Chico (Stone, 1928); Lago Frío (Pergolani de Costa 1975); Parque Nacional Los Glaciares, Parque Nacional Perito Moreno (Chebez et al., 1998); Parque Nacional Los Glaciares (Sección Centro) (Imberti, 2005); Parque Nacional Perito Moreno, Meseta Lago Buenos Aires, Parque Nacional Los Glaciares, Estancia La Soledad, Estancia La Anita (Di Giácomo et al., 2005).

Museum specimens. Lago Argentino, Lago Frío, 1 young, 23 Jan 1959, col. P. Miles, MACN.
Field observations. Parque Nacional Perito Moreno, Parque Nacional Los Glaciares, Monumento Natural Bosques Petrificados, Estancia Cóndor, Lago Posadas, Estancia Rincón de los Morros, Estancia La Angostura, Estancia Rupai Pacha (Imberti).

\section{Lessonia rufa}

Literature. Río Coig, Ría Coig (Stone, 1928); Puerto Deseado (Zapata, 1967); Caleta Olivia, Mazarredo (Zapata, 1969); Laguna Los Escarchados (Lange, 1981); Puerto Deseado, Ría Deseado, Puerto Santa Cruz (Humphrey et al., 1985); Reserva de Vida Silvestre Los Escarchados (Erize, 1983); Paso Gregores, La Esperanza (de la Peña, 1986, 2005); Estancia El Cuadro, Monumento Natural Bosques Petrificados (De Lucca \& Saggese, 1992); Monumento Natural Bosques Petrificados, Parque Nacional Los Glaciares, Parque Nacional Perito Moreno (Chebez et al., 1998); Laguna Nimez (Imberti \& Albrieu, 2001); Parque Nacional Los Glaciares (Sección Sur) (Imberti, 2005).

Museum specimens. Bahía del Fondo, 1 M, 28 Dec 1921, col. A. Merkle, MLP; Estancia El Puma, 1 M, 20 Jan 2000, CFA; Lago Argentino, Lago Roca, 1 M, 26 Jan 1959, col. P. Miles, MACN.

Field observations. Parque Nacional Perito Moreno, Parque Nacional Los Glaciares, Monumento Natural Bosques Petrificados, Estancia Rincón de los Morros, Cabo Vírgenes, Río Gallegos, Ría Gallegos, Estancia Rupai Pacha, Estancia 9 de Julio, Estancia Pali Aike, Laguna Los Escarchados, Puerto Bandera, Estancia Bella Vista Bitsch, Estancia La Soledad, Estancia Sofía, Ría Coig, El Roda, Estancia La Angostura, Monte León, Koluel Kayke (Imberti).

\section{Hymenops perspicillatus andinus}

Literature. Caprek-aik (Burmeister, 1890); Isla Pavón, Lago Viedma, Perito Moreno (Chebez et al., 1988); Estancia El Cuadro, Monumento Natural Bosques Petrificados (De Lucca \& Saggese, 1992); Puerto Bandera (Iglesias \& Pérez, 1998); Monumento Natural Bosques Petrificados, Parque Nacional Los Glaciares, (Chebez et al., 1998); Laguna Nimez (Imberti \& Albrieu, 2001); Estancia La Angostura, Laguna Nimez, Lago Posadas, El Pluma, Estancia La Soledad, Estancia María Aike, Parque Nacional Los Glaciares, Monumento Nacional Bosques Petrificados (Imberti, 2003); Estancia La Angostura, Bahía del Túnel, Puerto Bandera, Estancia Anita, Estancia Cristina (Imberti, 2005); Río Chico (Coconier, 2005).

Field observations. Monumento Natural Bosques Petrificados, Lago Posadas, Puerto 
Bandera, Estancia La Soledad, Laguna Nimez, El Pluma, Estancia La Angostura, Koluel Kayke (Imberti).

\section{Tyrannus savana savana}

Field observations. Estancia Rupai Pacha (Sturzenbaum pers. comm.).

\section{Tyrannus tyrannus}

Field observations. Parque Nacional Monte León (Militello y Schieda pers. comm.)

\section{Pitangus sulphuratus argentinus}

Field observations. Caleta Olivia, Las Heras (Imberti).

\section{COTINGIDAE}

\section{Phytotoma rutila rutila}

Field observations. Monumento Natural Bosques Petrificados (Zoratti pers. comm.).

\section{Phytotoma rara}

Literature. Lago Argentino, Lago Frío, Lago San Martín (Navas, 1965); Río Bote, Parque Nacional Los Glaciares (Chebez et al., 1988); Parque Nacional Los Glaciares, Parque Nacional Perito Moreno (Chebez et al., 1998); Los Antiguos (Earnshaw \& Earnshaw, 2000); Glaciar Perito Moreno (Imberti, 2005); Parque Nacional Perito Moreno, Laguna Nimez, Lago Argentino, Parque Nacional Los Glaciares, Estancia La Soledad, Estancia La Anita (Di Giácomo et al., 2005).

Museum specimens. Lago Argentino, Lago Frío, 2 M, 14 and 16 Jan 1959, col. P. Miles, MACN.

Field observations. Parque Nacional Perito Moreno, Parque Nacional Los Glaciares, Río La Leona, Parque Nacional Monte León, Estancia Tucu Tucu (Imberti).

\section{HIRUNDINIDAE}

\section{Progne modesta elegans}

Literature. Punta Maqueda (Zapata, 1969); Puerto Deseado (Humphrey et al., 1985); Estancia El Cuadro, Monumento Natural Bosques Petrificados (De Lucca \& Saggese, 1992); Estancia El Cuadro (De Lucca et al., 1993, Saggese \& De Lucca, 2001); Bahía Laura, Monumento Nacional Bosques Petrificados, Valle de Río Deseado, Koluel Kayke (Imberti, 2003).

Field observations. Monumento Natural Bosques Petrificados, Estancia María Aike, Perito Moreno, Las Heras, Caleta Olivia (Imberti).

\section{Progne tapera fusca}

Literature. Cabo Vírgenes (Nores \& Yzurieta, 1995).

\section{Tachycineta meyeni}

Literature. Laguna Los Escarchados (Lange, 1981); Reserva de Vida Silvestre Los Escarchados (Erize, 1983); Puerto Deseado, Ría Deseado, Río Deseado, Río Santa Cruz (Humphrey et al., 1985); Río Turbio (Fraga \& Narosky, 1985; de la Peña 1987, 2005); Estancia El Cuadro (De Lucca \& Saggese, 1992); Monumento Natural Bosques Petrificados, Parque Nacional Los Glaciares, Parque Nacional Perito Moreno (Chebez et al., 1998); Laguna Nimez (Imberti \& Albrieu, 2001); Lago Rico, Lago Roca (Imberti, 2005).

Museum specimens. Cancha Carrera, 1 F ?, 3 Feb 1976, col. M. Rumboll; Lago Argentino, Lago Frío, 1 M, 22 Jan 1959, col. P. Miles, MACN.

Field observations. Parque Nacional Perito Moreno, Parque Nacional Los Glaciares, Monumento Natural Bosques Petrificados, Lago Posadas, Estancia Rincón de los Morros, Bahía Redonda, Laguna Nimez, Estancia Rupai Pacha, Estancia 9 de Julio, Cabo Vírgenes, Laguna Los Escarchados, Puerto Bandera, Estancia La Soledad, Ría Coig, Estancia Pali Aike, Estancia Sofía, Estancia Bella Vista Bitsch, Estancia Bella Vista, Estancia La Angostura, Monte León, Koluel Kayke (Imberti).

\section{Notiochelidon cyanoleuca patagonica}

Literature. Bahía Lángara, Punta Maqueda (Zapata, 1969); Laguna Los Escarchados (Lange, 1981); Reserva de Vida Silvestre Los Escarchados (Erize, 1983); Puerto Deseado, Río Deseado, Puerto Santa Cruz, Río Santa Cruz (Humphrey et al., 1985); Monumento Natural Bosques Petrificados, Parque Nacional Los Glaciares, Parque Nacional Perito Moreno (Chebez et al., 1998); Laguna Nimez (Imberti \& Albrieu, 2001); Lago Rico, Lago Roca (Imberti, 2005).

Field observations. Parque Nacional Perito Moreno, Parque Nacional Los Glaciares, Monumento Natural Bosques Petrificados, Estancia Rincón de los Morros, Estancia Pali Aike, Estancia Rupai Pacha, Estancia 9 de Julio, Estancia Cabo Buen Tiempo, Cabo Vírgenes, Laguna Los Escarchados, Monte León, El Pluma, Estancia La Angostura, Monte León, Koluel Kayke (Imberti).

\section{Riparia riparia riparia}

Literature. Lago Rico, Lago Roca (Imberti, 2005). 


\section{Petrochelidon pyrrhonota pyrrhonota}

Literature. Puerto Deseado (Humphrey \& Bridge, 1970).

Field observations. Comandante Luis Piedra Buena (Earnshaw pers. comm.); Estancia La Angelina, Cabo Vírgenes (Imberti).

\section{Hirundo rustica erythrogaster}

Literature. Puerto Deseado, Puerto Coig (Humphrey \& Bridge, 1970); Estancia El Cuadro (De Lucca \& Saggese, 1992); Monumento Natural Bosques Petrificados (Chebez et al., 1998); Río Gallegos (Babarskas \& Chebez, 1999); Lago Roca, Puerto Bandera (Imberti, 2005); Cabo Vírgenes (Imberti unpubl. a); Estancia La Angostura (Imberti unpubl. b).

Field observations. Monumento Natural Bosques Petrificados, Estancia El Cóndor, Estancia Las Horquetas, Cabo Vírgenes, Estancia La Angostura (Imberti); Monte León, Isla Pavón (Earnshaw pers. comm.).

\section{TROGLODYTIDAE}

\section{Cistothorus platensis hornensis}

Literature. Puerto Bandera (Iglesias \& Pérez, 1998); Parque Nacional Los Glaciares (Chebez et al., 1998); Laguna Nimez (Imberti \& Albrieu, 2001); Bahía del Túnel, Estancia San José, Estancia Cristina, Puerto Bandera, Estancia Anita, Lago Roca (Imberti, 2005); Estancia El Cóndor (Coconier, 2005).

Field observations. Parque Nacional Los Glaciares, Cabo Vírgenes, Estancia La Angostura, El Zurdo, El Pluma, Laguna Nimez (Imberti).

\section{Troglodytes aedon chilensis}

Literature. Río Coig, Ría Coig, Río Chico, Cabo Buen Tiempo (Stone 1928); Puerto Deseado (Zapata, 1967; Humphrey et al., 1985); Laguna Los Escarchados (Lange, 1981); Estancia El Cuadro (De Lucca \& Saggese, 1992); Monumento Natural Bosques Petrificados, Parque Nacional Los Glaciares, Parque Nacional Perito Moreno (Chebez et al., 1998); Laguna Nimez (Imberti \& Albrieu, 2001); Parque Nacional Los Glaciares (Imberti 2005).

Museum specimens. Lago Argentino, Cerro Mayo, 1 M, 6 Feb 1959, col. P. Miles; Río Gallegos, $1 \mathrm{M}, 28$ Apr 1974, col. M. Rumboll, MACN.

Field observations. Parque Nacional Perito Moreno, Parque Nacional Los Glaciares, Monumento Natural Bosques Petrificados, Estancia Rincón de los Morros, Estancia Pali Aike, Estancia Rupai Pacha, Estancia 9 de Julio, Estancia Cabo Buen Tiempo, Cabo Vírgenes,
Lago Posadas, Estancia Cóndor, Río Gallegos, Ría Gallegos, Puerto Bandera, Estancia La Soledad, Lago del Desierto, Estancia La Angostura, Ría Coig, Punta Loyola, Estancia La Alice, Estancia El Cóndor, Ría Santa Cruz, Bahía San Julián, Estancia Bella Vista, Monte León, Koluel Kayke (Imberti).

\section{TURDIDAE}

\section{Turdus falcklandii magellanicus}

Literature. Cabo Vírgenes, Río Shehuén, Valle del Río Bote, Cuevas de Gualicho, El Calafate, Estancia Las Tunas (Chebez et al., 1988); Estancia El Cuadro (De Lucca \& Saggese, 1992); Monumento Natural Bosques Petrificados, Parque Nacional Los Glaciares, Parque Nacional Perito Moreno (Chebez et al., 1998); Los Antiguos (Earnshaw \& Earnshaw, 2000); Laguna Nimez (Imberti \& Albrieu, 2001); Parque Nacional Los Glaciares (Imberti, 2005); Estancia La Angostura (Imberti unpubl. b).

Museum specimens. Estancia El Puma, 1 F, 16 Jan 2000, CFA; Lago Argentino, brazo Sud, 1 F, 12 Jan 1959, col. P. Miles, MACN.

Field observations. Parque Nacional Perito Moreno, Parque Nacional Los Glaciares, Monumento Natural Bosques Petrificados, Estancia Rincón de los Morros, Estancia Rupai Pacha, Estancia Cabo Buen Tiempo, Cabo Vírgenes, Lago Posadas, Río Gallegos, Estancia Cóndor, Estancia El Cóndor, Punta Loyola, El Zurdo, Estancia Glencross, Estancia Morro Chico, Comandante Luis Piedra Buena, El Roda, Estancia Bella Vista, Monte León, Koluel Kayke (Imberti).

\section{MIMIDAE}

\section{Mimus patagonicus}

Literature. Río Chico, Caprek-aik (Burmeister, 1890); Isla Pavón (Salvadori, 1900); Santa Cruz, Río Chico (Stone, 1928); Puerto Deseado (Zapata, 1967); Puerto Santa Cruz, Monte León (Humphrey et al., 1985); Estancia El Cuadro, Monumento Natural Bosques Petrificados (De Lucca \& Saggese, 1992); Monumento Natural Bosques Petrificados, Parque Nacional Perito Moreno (Chebez et al., 1998); Laguna Nimez (Imberti \& Albrieu, 2001); Parque Nacional Los Glaciares (Sección Sur), Bahía del Túnel, El Chaltén (Imberti, 2005).

Museum specimens. Estancia El Puma, $1 \mathrm{M}$, 17 Jan 2000, CFA; Lago Argentino, Punta Bandera, 1 M, 21 Feb 1959, col. P. Miles, MACN. Field observations. Parque Nacional Perito Moreno, Parque Nacional Los Glaciares, 
Monumento Natural Bosques Petrificados, Estancia Rupai Pacha, Estancia Cabo Buen Tiempo, Cabo Vírgenes, Lago Posadas, Río Bote, Estancia La Soledad, Laguna Nimez, Monte León, Estancia Moy Aike Chico, Ría Coig, Ría Santa Cruz, Estancia Bella Vista, Estancia La Angostura, Koluel Kayke (Imberti).

MOTACILLIDAE

\section{Anthus correndera chilensis}

Literature. Chonque Aike (Burmeister, 1890); Río Coig, Cabo Buen Tiempo (Stone 1928); Mazarredo (Zapata, 1969); Estancia El Cuadro (De Lucca \& Saggese, 1992); Monumento Natural Bosques Petrificados, Parque Nacional Los Glaciares, Parque Nacional Perito Moreno (Chebez et al., 1998); Laguna Nimez (Imberti \& Albrieu, 2001); Parque Nacional Los Glaciares (Sección Sur) (Imberti, 2005).

Museum specimens. Lago Argentino, Lago Roca, 1 M, 26 Jan 1959, col. P. Miles, MACN.

Field observations. Parque Nacional Perito Moreno, Parque Nacional Los Glaciares, Monumento Natural Bosques Petrificados, Estancia Rincón de los Morros, Estancia Rupai Pacha, Cabo Vírgenes, El Zurdo, Lago Posadas, Río Gallegos, Laguna Nimez, Estancia Sofía, El Roda, Estancia María Aike, Estancia Bella Vista, Estancia La Angostura, Monte León, Koluel Kayke (Imberti).

\section{Anthus hellmayri dabbenei}

Literature. Isla Pavón (Chebez et al., 1988); Parque Nacional Los Glaciares (Chebez et al., 1998); Laguna Nimez (Imberti \& Albrieu, 2001); Bahía del Túnel (Imberti, 2005).

Field observations. Parque Nacional Los Glaciares, Estancia Rincón de los Morros, Estancia Rupai Pacha, Cabo Vírgenes, Estancia 9 de Julio, Ría Gallegos, Estancia Bella Vista Bitsch (Imberti).

\section{EMBERIZIDAE}

\section{Phrygilus gayi caniceps}

Literature. Aguada Grande (Dabbene, 1933); Lago San Martín (Radboone, 1935); Santa Cruz, Río Gallegos, Ría Coig, Cabo Buen Tiempo (Hellmayr, 1938); Puerto Deseado (Zapata, 1967); Cabo Blanco (Zapata, 1969); Río Turbio (de la Peña, 1987, 2005); Estancia El Cuadro, Monumento Natural Bosques Petrificados (De Lucca \& Saggese, 1992); Monumento Natural Bosques Petrificados, Parque Nacional Los Glaciares, Parque Nacional Perito Moreno (Chebez et al., 1998); Laguna Nimez (Imberti \& Albrieu, 2001); Parque Nacional Los Glaciares
(Sección Norte), Lago Viedma, El Chaltén, Bahía del Túnel (Imberti, 2005); Estancia El Cóndor (Coconier, 2005); Río Gallegos, Río Coig, Meseta Lago Strobel, Río Chico, Monumento Natural Bosques Petrificados, Estancia El Cuadro, Parque Nacional Perito Moreno, Meseta Lago Buenos Aires, El Zurdo, Laguna Nimez, Lago Argentino, Meseta del Asador, Parque Nacional Los Glaciares, Estancia La Soledad, Estancia La Anita, Estancia El Cóndor, Bahía San Julián (Di Giácomo et al., 2005).

Museum specimens. El Calafate, $70 \mathrm{~km}$ east, 1 F ?, 27 Apr 1974, col. M. Rumboll, MACN; Estancia El Puma, $1 \mathrm{M}$ and $1 \mathrm{~F}$, 19 Jan 2000, CFA; Laguna Azul, 2 F, 3 Feb 1972, col. A. Cicchino; Los Antiguos, Lago Buenos Aires, 1 M, 11 Sep 1983, col. O. Padín, MLP; Puerto Deseado, 1 F ?, 7 Aug 1961, col. A. Zapata; Río Gallegos, 1 M, 1 May 1974, col. M. Rumboll; Río Santa Cruz, ruta 3, 1 M, 4 May 1974, col. M. Rumboll, MACN.

Field observations. Parque Nacional Perito Moreno, Parque Nacional Los Glaciares, Monumento Natural Bosques Petrificados, Estancia Rincón de los Morros, Estancia Rupai Pacha, Cabo Vírgenes, El Zurdo, Lago Posadas, Estancia Sofía, Estancia Bella Vista, Lago Argentino, Río Bote, Estancia Cabo Buen Tiempo, Ría Coig, Monte León, Estancia Cóndor, Estancia La Alice, Estancia El Cóndor, Estancia Morro Chico, Estancia La Carlota, Punta Loyola, Estancia Los Luises, Estancia La Angostura, Koluel Kayke (Imberti).

\section{Phrygilus patagonicus}

Literature. Cabo Buen Tiempo, Ría Coig, Santa Cruz (Stone, 1928); Cabo Vírgenes, Cañadón Gapp (Chebez et al., 1988); Monumento Natural Bosques Petrificados, Parque Nacional Los Glaciares, Parque Nacional Perito Moreno (Chebez et al., 1998); Río Turbio (de la Peña, 2005); Parque Nacional Los Glaciares (Imberti, 2005).

Museum specimens. Lago Argentino, brazo Sud, 1 M, 12 Jan 1959; Lago Argentino, Lago Onelli, 1 M, 29 Dec 1958, col. P. Miles, MACN.

Field observations. Parque Nacional Perito Moreno, Parque Nacional Los Glaciares, Monumento Natural Bosques Petrificados, Estancia Rupai Pacha, Cabo Vírgenes, Estancia Cóndor (Imberti).

\section{Phrygilus fruticeti fruticeti}

Literature. Santa Cruz, Cañadón Misioneros (Oustalet, 1891); Río Chico, Río Ecker, Río Gallegos (Stone, 1928); Puerto Deseado (Zapata, 1967, Humphrey et al., 1985); Caleta Olivia (Zapata, 1969); Estancia Monte Dinero 
(Humphrey \& Bridge, 1970); Puerto Deseado, Estancia La Renania, Altos del Río Chico, Río Gallegos (Zapata \& Martínez, 1972); Estancia El Cuadro, Monumento Natural Bosques Petrificados (De Lucca \& Saggese, 1992); Monumento Natural Bosques Petrificados (Chebez et al.,1998); Monte Zeballos (Earnshaw \& Earnshaw, 2000); Laguna Nimez (Imberti \& Albrieu, 2001); Parque Nacional Los Glaciares (Sección Norte), Lago Roca, El Calafate (Imberti, 2005); Río Coig, Meseta Lago Strobel, Río Chico, Monumento Natural Bosques Petrificados, Estancia El Cuadro, Parque Nacional Perito Moreno, Meseta Lago Buenos Aires, Laguna Nimez, Lago Argentino, Meseta del Asador, Parque Nacional Los Glaciares, Estancia La Soledad, Estancia La Anita, Estancia El Cóndor, Bahía San Julián (Di Giácomo et al., 2005); Cabo Vírgenes (Imberti unpubl. a).

Museum specimens. Lago Argentino, Punta Bandera, 1 M and 1 F, 22 Feb 1959, col. P. Miles; Puerto Deseado, 1 F, 28 Nov 1961, col. A. Zapata, MACN.

Field observations. Monumento Natural Bosques Petrificados, Gobernador Gregores, El Calafate, Lago Posadas, Lago Argentino, Estancia La Angostura, Ría Coig, Bahía San Julián, Estancia El Cóndor, Monte León, Koluel Kayke (Imberti).

\section{Phrygilus unicolor unicolor}

Literature. Lago San Martín (Wetmore, 1926); Parque Nacional Los Glaciares, Parque Nacional Perito Moreno (Chebez et al., 1998);Monte Zeballos (Earnshaw \& Earnshaw, 2000); Lago Roca, Estancia Cristina, El Chaltén (Imberti, 2005); Meseta Lago Strobel, Parque Nacional Perito Moreno, Meseta Lago Buenos Aires, Laguna Nimez, Lago Argentino, Meseta del Asador, Parque Nacional Los Glaciares, Estancia La Soledad, Estancia La Anita (Di Giácomo et al., 2005); Estancia La Angostura (Imberti unpubl. b).

Museum specimens. Lago Argentino, brazo Sud, 1 M, 14 Jan 1959; Lago Argentino, Lago Frío, 1 M ?, 14 Jan 1959, col. P. Miles, MACN.

Field observations. Parque Nacional Perito Moreno, Parque Nacional Los Glaciares, Monte León (Imberti).

\section{Phrygilus carbonarius}

Field observations. Monumento Natural Bosques Petrificados (Zancaner pers. com.).

\section{Melanodera melanodera princetoniana}

Literature. Cheike (Scott, 1900b; Stone, 1928); Aguada Grande (Pozzi, 1923); Río Coig (Wetmore, 1926; Dabbene, 1933); Parque Nacional Los
Glaciares, Parque Nacional Perito Moreno (Chebez et al., 1998);El Zurdo, Estancia Sofía, Estancia Buitreras, Laguna Travesía, Laguna Los Escarchados (Imberti, 2003); Lago Roca (Imberti, 2005); Río Gallegos, El Zurdo, Parque Nacional Los Glaciares, Estancia El Cóndor (Coconier, 2005); Río Gallegos, Parque Nacional Perito Moreno, El Zurdo, Parque Nacional Los Glaciares, Estancia La Soledad, Estancia La Anita, Estancia El Cóndor (Di Giácomo et al., 2005).

Museum specimens. Aguada Grande, Río Santa Cruz, 2 M, 6 and 11 May 1923, col. A. Pozzi, MACN.

Field observations. Parque Nacional Los Glaciares, Estancia Verdadera Argentina, Cabo Vírgenes, El Zurdo, Estancia El Cóndor, Estancia La Carlota, Estancia Buitreras, El Roda, Estancia Monte Dinero, Estancia Sofía (Imberti).

\section{Melanodera xanthogramma barrosi}

Literature. Lago Viedma (Wetmore, 1926); Lago Viedma, Lago San Martín (Zotta, 1940); Río Centinela, Río Gallegos (Chebez et al., 1988); Parque Nacional Perito Moreno (Chebez et al., 1998); Parque Nacional Los Glaciares (Imberti, 2005); El Zurdo (Coconier, 2005).

Museum specimens. Lago San Martín, 1 M, Jan 1936, MACN.

Field observations. Parque Nacional Perito Moreno, Parque Nacional Los Glaciares, Estancia Verdadera Argentina, Estancia Sofía, El Zurdo, Estancia La Carlota (Imberti).

\section{Diuca diuca diuca}

Literature. Parque Nacional Los Glaciares (Chebez et al., 1998); Parque Nacional Los Glaciares, Lago Argentino, Lago Posadas, Estancia El Cóndor (Imberti, 2003); El Chaltén, Lago Roca, El Calafate (Imberti, 2005).

Field observations. Parque Nacional Los Glaciares, Lago Posadas (Imberti).

\section{Diuca diuca minor}

Literature. Cañadón Misioneros (Oustalet, 1891, Hellmayr, 1938); Monumento Nacional Bosques Petrificados, Río Bote, Estancia La Angostura, Estancia La Lucha, Estancia Morro Chico, Estancia La Cabaña, Gobernador Gregores (Imberti, 2003).

Field observations. Monumento Natural Bosques Petrificados, Estancia Cóndor, Río Bote, Estancia La Angostura, Estancia La Lucha, Estancia Morro Chico, Monte León, Koluel Kayke (Imberti).

\section{Sicalis auriventris}

Literature. Sierra Baguales (Imberti, 2003) 
Field observations. Estancia La Querencia, Río La Leona, Estancia Glencross, Río Turbio, Ruta Provincial 41 (Imberti).

\section{Sicalis lebruni}

Literature. Cañadón Misioneros (Oustalet, 1891, Hellmayr, 1938); Río Gallegos, Ría Coig (Wetmore, 1926); Santa Cruz, Río Gallegos (Stone, 1928); Estancia El Cuadro (De Lucca \& Saggese, 1992); Monumento Natural Bosques Petrificados, Parque Nacional Los Glaciares, Parque Nacional Perito Moreno (Chebez et al., 1998); Parque Nacional Los Glaciares (Sección Norte, Sección Centro), Lago Roca, Glaciar Perito Moreno (Imberti, 2005); Río Gallegos, Río Chico, Río Chico, Monumento Natural Bosques Petrificados, Estancia El Cuadro, Parque Nacional Perito Moreno, Meseta Lago Buenos Aires, El Zurdo, Laguna Nimez, Lago Argentino, Meseta del Asador, Parque Nacional Los Glaciares, Estancia La Soledad, Estancia La Anita, Estancia El Cóndor, Bahía San Julián (Di Giácomo et al., 2005); Estancia La Angostura (Imberti unpubl. b).

Field observations. Parque Nacional Los Glaciares, Parque Nacional Perito Moreno, Monumento Natural Bosques Petrificados, Estancia El Cóndor, Estancia Rupai Pacha, El Zurdo, Punta Loyola, Cabo Vírgenes, Estancia Pali Aike, Estancia Cabo Buen Tiempo, El Roda, Monte León, Koluel Kayke (Imberti).

\section{Sicalis luteola luteiventris}

Literature. El Pluma (Imberti 2003); Río Chico (Di Giácomo et al., 2005).

Field observations. El Pluma, Valle Río Deseado, Estancia Las Tunas (Imberti).

\section{Zonotrichia capensis australis}

Literature. Cabo Curioso (Burmeister, 1890); Cabo Buen Tiempo, Monte Tigre (Stone, 1928); Puerto Deseado (Zapata, 1967; Daciuk, 1977); Laguna Los Escarchados (Lange, 1981); Reserva de Vida Silvestre Los Escarchados (Erize, 1983); Puerto Deseado, Puerto Santa Cruz (Humphrey et al., 1985); Estancia El Cuadro, Monumento Natural Bosques Petrificados (De Lucca \& Saggese, 1992); Monumento Natural Bosques Petrificados, Parque Nacional Los Glaciares, Parque Nacional Perito Moreno (Chebez et al., 1998); Laguna Nimez (Imberti \& Albrieu, 2001); La Manchuria, Aguada Grande, Río Gallegos, Lago Argentino, Cerro Bayo, Lago San Martín (Piloni, 2002 [2004]); Parque Nacional Los Glaciares (Imberti, 2005).

Museum specimens. Lago Argentino, Cerro Mayo, 1 F, 11 Feb 1959, col. P. Miles; Río Gallegos, 1 M, 4 Feb 1971, col. A. Cicchino, MLP, 1 M, 1
May 1974, col. M. Rumboll, MACN.

Field observations. Parque Nacional Los Glaciares, Parque Nacional Perito Moreno, Monumento Natural Bosques Petrificados, Estancia El Cóndor, Estancia Cóndor, Estancia Rupai Pacha, El Zurdo, Punta Loyola, Cabo Vírgenes, Estancia Pali Aike, Estancia Cabo Buen Tiempo, Lago Posadas, Río Gallegos, Ría Gallegos, Estancia 9 de Julio, Río Bote, Puerto Bandera, Estancia La Soledad, Laguna Nimez, Estancia Rincón de los Morros, Lago del Desierto, Estancia La Angostura, Ría Coig, Río Coig, Laguna Azul, Estancia Sofía, Estancia Bella Vista Bitsch, Monte León, Estancia Glencross, Estancia La Alice, Santa Cruz, Ría Santa Cruz, Bahía San Julián, Estancia María Aike, El Pluma, El Roda, Koluel Kayke (Imberti).

\section{ICTERIDAE}

\section{Agelaius thilius petersi}

Literature. Estancia Alta Vista, Punta Bandera, Perito Moreno (Chebez et al., 1988); Estancia La Angostura, Río Chico (Mazar Barnett et al., 1998); Puerto Bandera (Iglesias \& Pérez, 1998); Monumento Natural Bosques Petrificados, Parque Nacional Los Glaciares (Chebez et al., 1998); Laguna Nimez (Imberti \& Albrieu, 2001); El Pluma, Laguna Nimez, Lago Posadas, Estancia La Angostura, Estancia La Soledad, Estancia 9 de Julio, Estancia San Lorenzo, Estancia La Alice, Estancia María Aike, Parque Nacional Los Glaciares, Monumento Nacional Bosques Petrificados (Imberti 2003); Bahía del Túnel, Estancia San José, Estancia Cristina, Puerto Bandera, Estancia Anita, Estancia San Lorenzo (Imberti, 2005); Río Chico (Coconier, 2005).

Field observations. Monumento Natural Bosques Petrificados, Estancia El Cóndor, Estancia Rupai Pacha, El Zurdo, Estancia Pali Aike, Estancia La Angostura, Estancia San Lorenzo, Parque Nacional Los Glaciares, Laguna Nimez, Laguna Balton (Imberti).

\section{Curaeus curaeus curaeus}

Literature. Puerto Deseado (Zapata, 1967); Parque Nacional Los Glaciares, Parque Nacional Perito Moreno (Chebez et al., 1998); Parque Nacional Los Glaciares (Imberti, 2005); Estancia La Angostura (Imberti unpubl. b).

Field observations. Parque Nacional Los Glaciares, Parque Nacional Perito Moreno, Estancia Cóndor, Estancia Glencross, Estancia La Alice, Lago Posadas, Laguna Nimez, Estancia Rincón de los Morros, Estancia Tucu Tucu (Imberti).

\section{Molothrus bonariensis bonariensis}

Literature. Río Deseado (Burmeister, 1890); 
Parque Nacional Perito Moreno (Chebez et al., 1998); Parque Nacional Perito Moreno, El Pluma (Imberti, 2003).

Field observations. El Pluma, Koluel Kayke, Parque Nacional Los Glaciares (Imberti); Parque Nacional Perito Moreno (Falcone pers. comm.).

\section{Sturnella loyca loyca}

Literature. Caprek-aik, Emelek-aike (Burmeister, 1890); Cañadón Misioneros, Santa Cruz (Oustalet, 1891); Isla Pavón (Salvadori, 1900); Río Coig (Wetmore, 1926); Estancia Pali Aike, Río Gallegos (Stone, 1928); Aguada Grande (Zotta, 1937); Río Gallegos (Olrog, 1948); Puerto Deseado (Zapata, 1967, de la Peña, 1987, 2005); Punta Bauzá, Mazarredo (Zapata, 1969); Puerto Deseado, Puerto Santa Cruz (Humphrey et al., 1985); Estancia El Cuadro, Monumento Natural Bosques Petrificados (De Lucca \& Saggese, 1992); Monumento Natural Bosques Petrificados, Parque Nacional Los Glaciares, Parque Nacional Perito Moreno (Chebez et al., 1998); Laguna Nimez (Imberti \& Albrieu, 2001); Lago Roca, Sierra de los Cristales, El Chaltén (Imberti, 2005). Museum specimens. Aguada Grande, Depto. Corpen Aike, 1 M, 4 May 1923, col. A. Pozzi, MACN. Estancia La Renania, 1 F, 24 Apr 1949, col. J. Becerra, MLP. Estancia Roca Blanca, 1 M and 2 F, 23 Dec 1960, 11 and 17 Jan 1961, col. A. Budin, FML. Lago Frío, Lago Argentino, $1 \mathrm{M}$ and 1 F, 16 Jan 1959, col. P. Miles. Lago San Martín, Depto. Lago Argentino, 1 M, 8 Aug 1932, col. S. Radboone, MACN.

Field observations. Parque Nacional Los Glaciares, Parque Nacional Perito Moreno, Monumento Natural Bosques Petrificados, Estancia El Cóndor, Estancia Cóndor, Estancia Rupai Pacha, El Zurdo, Punta Loyola, Cabo Vírgenes, Estancia Pali Aike, Estancia Cabo Buen Tiempo, Lago Posadas, Río Gallegos, Ría Gallegos, Estancia Rincón de los Morros, Estancia La Angostura, Ría Coig, Estancia Bella Vista Bitsch, Monte León, Ría Santa Cruz, Bahía San Julián, Estancia María Aike, Estancia San Lorenzo, Comandante Luis Piedra Buena, El Calafate, El Roda, Koluel Kayke (Imberti).

\section{FRINGILLIDAE}

\section{Carduelis barbata}

Literature. Estancia Las Tunas (Chebez et al., 1988); Monumento Natural Bosques Petrificados, Parque Nacional Los Glaciares, Parque Nacional Perito Moreno (Chebez et al., 1998); Estancia El Cóndor, Bahía Posesión (Cadierno \& Amorós, 1999); Monte Zeballos (Earnshaw \& Earnshaw, 2000); Laguna Nimez (Imberti \&
Albrieu, 2001); Parque Nacional Los Glaciares (Imberti, 2005); Estancia La Angostura (Imberti unpubl. b).

Museum specimens. Lago Argentino, brazo Sud, 1 F, 12 Jan 1959, col. P. Miles, MACN; Los Antiguos, Lago Buenos Aires, 1 M, 11 Sep 1983, col. O. Padín, MLP.

Field observations. Parque Nacional Los Glaciares, Parque Nacional Perito Moreno, Monumento Natural Bosques Petrificados, Estancia El Cóndor, Estancia Cóndor, Estancia Rupai Pacha, El Zurdo, Cabo Vírgenes, Río Gallegos, Río Bote, Estancia Rincón de los Morros, Estancia Glencross, Ría Santa Cruz, Estancia Las Tunas, Estancia La Carreta, Monte León, Koluel Kayke (Imberti).

\section{PASSERIDAE}

\section{Passer domesticus}

Literature. Puerto Deseado (Zapata, 1967); Puerto Deseado, Puerto Santa Cruz (Humphrey et al., 1985); Estancia El Cuadro (De Lucca \& Saggese, 1992); Monumento Natural Bosques Petrificados, Parque Nacional Los Glaciares (Chebez et al., 1998); Laguna Nimez (Imberti \& Albrieu, 2001); Puerto Bandera, El Chaltén (Imberti, 2005).

Field observations. Parque Nacional Los Glaciares, Monumento Natural Bosques Petrificados, Estancia Rupai Pacha, Cabo Vírgenes, Estancia Cabo Buen Tiempo, Río Gallegos, Río Bote, Estancia Rincón de los Morros, Estancia La Angostura, Santa Cruz, Estancia Morro Chico, Comandante Luis Piedra Buena, Estancia Tapi Aike, Monte León, Koluel Kayke (Imberti).

\section{CONCLUSIONS}

The study of literature, museum specimens and field observations permitted us establish that Tyrannidae (21 spp), Furnariidae (19 spp), Emberizidae (12 spp), Hirundinidae (7 spp) and Icteridae ( $4 \mathrm{spp})$ are the best represented families in Santa Cruz Province.

Based on field observations, the first precise localities are presented for Tyrannus savana, Tyrannus tyrannus, Pitangus sulphuratus, Phytotoma rutila and Phrygilus carbonarius, and the southernmost record for Geositta rufipennis. New localities are provided for 64 species.

\section{ACKNOWLEDGEDMENTS}

For allowing us access to, or supplying specimen data on the collection in their care, we extend thanks to Jorge R. Navas, Pablo Tubaro and 
Yolanda Davies (MACN), and Ada L. Echevarría (FML). The following persons provided information on their own field observations: C. Albrieu, M. Díaz, A. Earnshaw, A. Falcone, S. Ferrari, M. McNamara, E. Militello, P. and S. Sturzenbaum, L. Bernacci, M. Bertinat, J. Schieda, V. Sotelo, J. Zancaner and C. Zoratti. We thank Cecilia Gorretta for the map and Lucas Marti for the English translation.

Appendix 1 - Localities that had not been cited in the paper about non Passeriformes (Darrieu et al. 2008).

19 - Cabo Curioso $\left(49^{\circ} 10^{\prime} \mathrm{S}-67^{\circ} 37^{\prime} \mathrm{W}\right)$ 14 - Cabo Dañoso (48 $50^{\circ} \mathrm{S}-67^{\circ} 13^{\prime} \mathrm{W}$ ) 21 - Cerro Torre (ca. $49^{\circ} 10^{\prime} \mathrm{S}-73^{\circ} 10^{\prime} \mathrm{W}$ ) 30 - Cuevas de Gualicho (ca. 5020' S - 72 $18^{\prime}$ W) 36 - Ea. Coy Aike (51 $\left.08^{\circ} \mathrm{S}-69^{\circ} 29^{\prime} \mathrm{W}\right)$

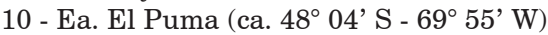
12 - Ea. La Cabaña $\left(48^{\circ} 34^{\prime} \mathrm{S}-70^{\circ} 29^{\prime} \mathrm{W}\right)$ 35 - Ea. Las Vegas (ca. $50^{\circ} 58^{\prime} \mathrm{S}-69^{\circ} 11^{\prime} \mathrm{W}$ ) 28 - Ea. La Unión (ca. $50^{\circ} 13^{\prime} \mathrm{S}-72^{\circ} 25^{\prime} \mathrm{W}$ ) 25 - Ea. Luz Divina ( $49^{\circ} 54^{\prime} \mathrm{S}-72^{\circ} 04^{\prime} \mathrm{W}$ ) 26 - Ea. María Cristina ( $\left.49^{\circ} 57^{\prime} \mathrm{S}-69^{\circ} 54^{\prime} \mathrm{W}\right)$ 18 - Ea. Rincón de los Toros (ca. $48^{\circ} 55^{\prime}$ S - 71 $10^{\circ}$ W) 22 - Ea. San José (ca. $49^{\circ} 27^{\prime}$ S - $72^{\circ} 52^{\prime}$ W)

15 - Ea. Tucu Tucu ( $\left.48^{\circ} 27^{\prime} \mathrm{S}-71^{\circ} 51^{\prime} \mathrm{W}\right)$

2- Gol-aik $\left(46^{\circ} 39^{\prime} \mathrm{S}-68^{\circ} 44^{\prime} \mathrm{W}\right)$

7 - Jaramillo ( $47^{\circ} 11^{\prime} \mathrm{S}$ - $67^{\circ} 09^{\prime} \mathrm{W}$ )

3 - Koluel Kayke (46 $43^{\circ}$ S - $\left.68^{\circ} 13^{\prime} \mathrm{W}\right)$

11 - La Manchuria (ca. $48^{\circ} 04^{\prime} \mathrm{S}-69^{\circ} 55^{\prime} \mathrm{W}$ )

6 - Lago Chico (ca. $47^{\circ} 11^{\prime} \mathrm{S}$ - $67^{\circ} 09^{\prime} \mathrm{W}$ )

32 - Lago Onelli (ca. $50^{\circ} 20^{\prime} \mathrm{S}-73^{\circ} 15^{\prime} \mathrm{W}$ )

13 - Lago Strobel (ca. $48^{\circ} 40^{\prime} \mathrm{S}-71^{\circ} 30^{\prime} \mathrm{W}$ )

24 - Laguna Balton $\left(49^{\circ} 25^{\prime} \mathrm{S}-71^{\circ} 42^{\prime} \mathrm{W}\right)$

17 - Meseta del Cardiel Chico (ca. $48^{\circ} 55^{\prime} \mathrm{S}-71^{\circ} 52^{\prime} \mathrm{W}$ )

1 - Monte Zeballos (ca. $46^{\circ} 41^{\prime} \mathrm{S}-71^{\circ} 37^{\prime} \mathrm{W}$ )

8 - Paso Gregores (47 $\left.48^{\prime} \mathrm{S}-66^{\circ} 35^{\prime} \mathrm{W}\right)$

5 - Paso Roballos $\left(47^{\circ} 05^{\prime} \mathrm{S}-71^{\circ} 50^{\prime} \mathrm{W}\right)$

16 - Puesto Ensenada (ca. $48^{\circ} 40^{\prime} \mathrm{S}-71^{\circ} 30^{\prime} \mathrm{W}$ )

20 - Puesto La Nana ( $48^{\circ} 56^{\prime} \mathrm{S}-72^{\circ} 39^{\prime} \mathrm{W}$ )

29 - Punta Avellaneda (ca. $50^{\circ} 13^{\prime} \mathrm{S}-72^{\circ} 25^{\prime} \mathrm{W}$ )

4 - Punta Bauzá ( $46^{\circ} 42^{\prime} \mathrm{S}$ - $67^{\circ} 09^{\prime} \mathrm{W}$ )

27 - Punta Cascajo (50 08' S - 68 $21^{\circ}$ ' W)

31 - Río Centinela $\left(50^{\circ} 19^{\prime} \mathrm{S}-72^{\circ} 31^{\prime} \mathrm{W}\right)$

9 - Río Lista (= Ea. Río Lista) (48 $\left.07^{\prime} \mathrm{S}-71^{\circ} 48^{\prime} \mathrm{W}\right)$ 23 - Río Sheuhén o Chalía ( $49^{\circ} 35^{\prime} \mathrm{S}$ - $69^{\circ} 34^{\prime} \mathrm{W}$ )

Appendix 2 - Localities that had been cited in the paper about non Passeriformes (Darrieu et al. 2008).

Aguada Grande (= Ea. Aguada Grande) (50 15' S $\left.69^{\circ} 38^{\prime} \mathrm{W}\right)$

Bahía del Fondo (= Caleta del Fondo) $\left(46^{\circ} 06^{\prime} \mathrm{S}-67^{\circ}\right.$ $36^{\prime} \mathrm{W}$ )

Bahía del Túnel (ca. $20^{\prime} \mathrm{S}-72^{\circ} 55^{\prime} \mathrm{W}$ )

Bahía Lángara ( $\left.46^{\circ} 37^{\prime} \mathrm{S}-67^{\circ} 19^{\prime} \mathrm{W}\right)$

Bahía Laura $\left(48^{\circ} 21^{\prime}\right.$ S - $66^{\circ} 21^{\prime}$ W)
Bahía Posesión (ca. 52 $15^{\prime} \mathrm{S}-69^{\circ} 00^{\prime} \mathrm{W}$ )

Bahía Redonda (50 $10^{\circ}$ ' S - $\left.72^{\circ} 16^{\prime} \mathrm{W}\right)$

Bahía San Julián (ca. 18' S - 67 43’ W)

Bajada de Míguez $\left(50^{\circ} 20^{\prime} \mathrm{S}-71^{\circ} 31^{\prime} \mathrm{W}\right)$

Brazo Rico (ca. 13' S - 72 $25^{\prime}$ W)

Cabo Blanco ( $\left.47^{\circ} 12^{\prime} \mathrm{S}-65^{\circ} 45^{\prime} \mathrm{W}\right)$

Cabo Buen Tiempo (51 $31^{\circ}$ ' S - $68^{\circ} 57^{\prime} \mathrm{W}$ )

Cabo Vírgenes ( $\left.52^{\circ} 19^{\prime} \mathrm{S}-68^{\circ} 21^{\prime} \mathrm{W}\right)$

Caleta Olivia ( $\left.46^{\circ} 26^{\prime} \mathrm{S}-67^{\circ} 32^{\prime} \mathrm{W}\right)$

Cancha Carrera $\left(51^{\circ} 15^{\prime} \mathrm{S}-72^{\circ} 14^{\prime} \mathrm{W}\right)$

Cañadón Darwin (no localizada)

Cañadón Gapp (52 $\left.10^{\circ} \mathrm{S}-68^{\circ} 34^{\prime} \mathrm{W}\right)$

Cañadón Leones (no localizada)

Cañadón Misioneros (50 01' S - 68 $31^{\circ}$ W)

Caprek-aik $\left(46^{\circ} 01^{\prime} \mathrm{S}-69^{\circ} 20^{\prime} \mathrm{W}\right)$

Cerro Bayo (no localizada)

Cerro Mayo $\left(50^{\circ} 20^{\prime} \mathrm{S}-73^{\circ} 30^{\prime} \mathrm{W}\right)$

Cheike (posiblemente Ea. Chall Aike: $50^{\circ} 30^{\prime} \mathrm{S}-70^{\circ}$ 50 ' W)

Chonque Aike $\left(49^{\circ} 24^{\prime} \mathrm{S}-69^{\circ} 32^{\prime} \mathrm{W}\right)$

Comandante Luis Piedra Buena ( $49^{\circ} 59^{\prime} \mathrm{S}-68^{\circ} 54^{\prime}$ $\mathrm{W})$

Ea. 9 de Julio $\left(50^{\circ} 23^{\prime} \mathrm{S}-72^{\circ} 43^{\prime} \mathrm{W}\right)$

Ea. Alta Vista $\left(50^{\circ} 28^{\prime} \mathrm{S}-72^{\circ} 36^{\prime} \mathrm{W}\right)$

Ea. Anita (ca. $50^{\circ} 13^{\prime} \mathrm{S}-72^{\circ} 25^{\prime} \mathrm{W}$ )

Ea. Bella Vista (ca. $51^{\circ} 38^{\prime} \mathrm{S}-69^{\circ} 13^{\prime} \mathrm{W}$ )

Ea. Bella Vista Bitsch ( $51^{\circ} 50^{\prime} \mathrm{S}-69^{\circ} 33^{\prime} \mathrm{W}$ )

Ea. Buitreras $\left(51^{\circ} 44^{\prime} \mathrm{S}-70^{\circ} 08^{\prime} \mathrm{W}\right)$

Ea. Cabo Buen Tiempo (ca. $51^{\circ} 33^{\prime} \mathrm{S}-68^{\circ} 57^{\prime} \mathrm{W}$ )

Ea. Cóndor (52 $\left.09^{\prime} \mathrm{S}-69^{\circ} 04^{\prime} \mathrm{W}\right)$

Ea. Coy Inlet $\left(50^{\circ} 58^{\prime} \mathrm{S}-69^{\circ} 13^{\prime} \mathrm{W}\right)$

Ea. Cristina $\left(49^{\circ} 52^{\prime} \mathrm{S}-73^{\circ} 07^{\prime} \mathrm{W}\right)$

Ea. El Cóndor (= El Cóndor) (ca. $52^{\circ} 10^{\prime} \mathrm{S}-69^{\circ} 02^{\prime}$ W)

Ea. El Cuadro ( $\left.47^{\circ} 30^{\prime} \mathrm{S}-68^{\circ} 00^{\prime} \mathrm{W}\right)$

Ea. El Tranquilo (50 $\left.08^{\prime} \mathrm{S}-71^{\circ} 41^{\prime} \mathrm{W}\right)$

Ea. Glencross (51 $\left.50^{\prime} \mathrm{S}-71^{\circ} 33^{\prime} \mathrm{W}\right)$

Ea. Helsingfors (ca. $35^{\prime} \mathrm{S}-72^{\circ} 35^{\prime} \mathrm{W}$ )

Ea. La Alice $\left(50^{\circ} 20^{\prime} \mathrm{S}-70^{\circ} 32^{\prime} \mathrm{W}\right)$

Ea. La Angelina ( $\left.51^{\circ} 25^{\prime} \mathrm{S}-69^{\circ} 04^{\prime} \mathrm{W}\right)$

Ea. La Angostura ( $48^{\circ} 37^{\prime} \mathrm{S}-70^{\circ} 39^{\prime} \mathrm{W}$ )

Ea. La Carlota $\left(51^{\circ} 39^{\prime} \mathrm{S}-69^{\circ} 35^{\prime} \mathrm{W}\right)$

Ea. La Carreta $\left(48^{\circ} 57^{\prime} \mathrm{S}-70^{\circ} 12^{\prime} \mathrm{W}\right)$

Ea. La Lucha $\left(48^{\circ} 29^{\prime} \mathrm{S}-70^{\circ} 26^{\prime} \mathrm{W}\right)$

Ea. La Querencia $\left(47^{\circ} 01^{\prime} \mathrm{S}-67^{\circ} 24^{\prime} \mathrm{W}\right)$

Ea. La Quinta $\left(49^{\circ} 20^{\prime} \mathrm{S}-72^{\circ} 54^{\prime} \mathrm{W}\right)$

Ea. La Renania $\left(47^{\circ} 02^{\prime} \mathrm{S}-70^{\circ} 00^{\prime} \mathrm{W}\right)$

Ea. La Soledad $\left(50^{\circ} 20^{\prime} \mathrm{S}-72^{\circ} 47^{\prime} \mathrm{W}\right)$

Ea. Las Horquetas ( $\left.51^{\circ} 22^{\prime} \mathrm{S}-70^{\circ} 14^{\prime} \mathrm{W}\right)$

Ea. Las Tunas (ca. $48^{\circ} 55^{\prime} \mathrm{S}-71^{\circ} 10^{\prime} \mathrm{W}$ )

Ea. Los Luises $\left(50^{\circ} 41^{\prime} \mathrm{S}-69^{\circ} 29^{\prime} \mathrm{W}\right)$

Ea. María Aike ( $\left.46^{\circ} 41^{\prime} \mathrm{S}-69^{\circ} 39^{\prime} \mathrm{W}\right)$

Ea. Monte Dinero (ca. $52^{\circ} 20^{\prime} \mathrm{S}-68^{\circ} 35^{\prime} \mathrm{W}$ )

Ea. Morro Chico (51 $57^{\prime} \mathrm{S}-71^{\circ} 33^{\prime} \mathrm{W}$ )

Ea. Moy Aike Chico (51 $\left.01^{\circ} \mathrm{S}-69^{\circ} 40^{\prime} \mathrm{W}\right)$

Ea. Palermo Aike ( $51^{\circ} 41^{\prime} \mathrm{S}-69^{\circ} 43^{\prime} \mathrm{W}$ )

Ea. Pali Aike (51 $\left.58^{\prime} \mathrm{S}-69^{\circ} 42^{\prime} \mathrm{W}\right)$

Ea. Punta Loyola (ca. $51^{\circ} 38^{\prime} \mathrm{S}-69^{\circ} 05^{\prime} \mathrm{W}$ )

Ea. Rincón de los Morros ( $51^{\circ} 55^{\prime} \mathrm{S}-71^{\circ} 30^{\prime} \mathrm{W}$ )

Ea. Roca Blanca (ca. $48^{\circ} 40^{\prime} \mathrm{S}-68^{\circ} 50^{\prime} \mathrm{W}$ )

Ea. Rupai Pacha (51 $01^{\circ}$ S - $72^{\circ} 04^{\prime} \mathrm{W}$ )

Ea. San Lorenzo $\left(49^{\circ} 33^{\prime} \mathrm{S}-72^{\circ} 28^{\prime} \mathrm{W}\right)$

Ea. Sofía $\left(51^{\circ} 57^{\prime} \mathrm{S}-70^{\circ} 49^{\prime} \mathrm{W}\right)$

Ea. Verdadera Argentina ( $50^{\circ} 51^{\prime} \mathrm{S}-72^{\circ} 11^{\prime} \mathrm{W}$ ) 
El Calafate $\left(50^{\circ} 20^{\prime} \mathrm{S}-72^{\circ} 18^{\prime} \mathrm{W}\right)$

El Chaltén $\left(49^{\circ} 20^{\prime} \mathrm{S}-72^{\circ} 55^{\prime} \mathrm{W}\right)$

El Pluma $\left(46^{\circ} 30^{\prime} \mathrm{S}-70^{\circ} 00^{\prime} \mathrm{W}\right)$

El Roda (51 $11^{\circ}$ ' S - $\left.71^{\circ} 46^{\prime} \mathrm{W}\right)$

El Zurdo ( $\left.52^{\circ} 00^{\prime} \mathrm{S}-71^{\circ} 15^{\prime} \mathrm{W}\right)$

Emelk-aik $\left(49^{\circ} 50^{\prime} \mathrm{S}-68^{\circ} 37^{\prime} \mathrm{W}\right)$

Fitz Roy $\left(47^{\circ} 02^{\prime} \mathrm{S}-67^{\circ} 15^{\prime} \mathrm{W}\right)$

Glaciar Perito Moreno (ca. $50^{\circ} 30^{\prime} \mathrm{S}-73^{\circ} 15 \mathrm{~W}$ )

Gobernador Gregores (48 $43^{\prime} \mathrm{S}-70^{\circ} 14^{\prime} \mathrm{W}$ )

Isla Pavón (50 $\left.00^{\prime} \mathrm{S}-68^{\circ} 55^{\prime} \mathrm{W}\right)$

La Esperanza (ca. $51^{\circ} 20^{\prime} \mathrm{S}-72^{\circ} 05^{\prime} \mathrm{W}$ )

Lago Argentino (50 $13^{\prime} \mathrm{S}-72^{\circ} 25^{\prime} \mathrm{W}$ )

Lago Buenos Aires ( $46^{\circ} 30^{\prime} \mathrm{S}-72^{\circ} 00^{\prime} \mathrm{W}$ )

Lago del Desierto $\left(49^{\circ} 02^{\prime} \mathrm{S}-72^{\circ} 58^{\prime} \mathrm{W}\right)$

Lago Frío (ca. $50^{\circ} 20^{\prime} \mathrm{S}-72^{\circ} 18^{\prime} \mathrm{W}$ )

Lago Posadas $\left(47^{\circ} 27^{\prime} \mathrm{S}-71^{\circ} 51^{\prime} \mathrm{W}\right)$

Lago Rico (no localizada)

Lago Roca (ca. 50 $30^{\prime} \mathrm{S}-72^{\circ} 40^{\prime} \mathrm{W}$ )

Lago San Martín ( $48^{\circ} 52^{\prime} \mathrm{S}-72^{\circ} 40^{\prime} \mathrm{W}$ )

Lago Viedma $\left(49^{\circ} 35^{\prime} \mathrm{S}-72^{\circ} 35^{\prime} \mathrm{W}\right)$

Lagos del Furioso ( $47^{\circ} 28^{\prime} \mathrm{S}-71^{\circ} 47^{\prime} \mathrm{W}$ )

Laguna Azul (52 $04^{\prime}$ S - $69^{\circ} 34^{\prime} \mathrm{W}$ )

Laguna Los Escarchados (50 24' S - $\left.71^{\circ} 33^{\prime} \mathrm{W}\right)$

Laguna Nimez $\left(50^{\circ} 19^{\prime} \mathrm{S}-72^{\circ} 15^{\prime} \mathrm{W}\right)$

Laguna Travesía $\left(51^{\circ} 21^{\prime} \mathrm{S}-71^{\circ} 48^{\prime} \mathrm{W}\right)$

Las Heras ( $46^{\circ} 33^{\prime} \mathrm{S}-68^{\circ} 57^{\prime} \mathrm{W}$ )

Los Antiguos ( $46^{\circ} 33^{\prime} \mathrm{S}-71^{\circ} 37^{\prime} \mathrm{W}$ )

Mazarredo ( $\left.47^{\circ} 05^{\prime} \mathrm{S}-66^{\circ} 42^{\prime} \mathrm{W}\right)$

Meseta de las Vizcachas ( $\left.50^{\circ} 35^{\prime} \mathrm{S}-71^{\circ} 55^{\prime} \mathrm{W}\right)$

Meseta del Strobel (ca. $48^{\circ} 40^{\prime} \mathrm{S}-71^{\circ} 30^{\prime} \mathrm{W}$ )

Meseta El Moro (no localizada)

Meseta La Siberia (ca. $48^{\circ} 55^{\prime} \mathrm{S}-71^{\circ} 10^{\prime} \mathrm{W}$ )

Monte León (= Ea. Monte León) (ca. $50^{\circ} 14^{\prime} \mathrm{S}-68^{\circ}$ $55^{\prime} \mathrm{W}$ )

Monte Tigre (51 $\left.20^{\prime} \mathrm{S}-69^{\circ} 05^{\prime} \mathrm{W}\right)$

Monumento Natural Bosques Petrificados (47 $40^{\prime} \mathrm{S}$ $68^{\circ} 00^{\prime} \mathrm{W}$ )

Pampa del Asador (475' S - $71^{\circ} 20^{\prime} \mathrm{W}$ )

Parque Nacional Los Glaciares (ca. $50^{\circ} 00^{\prime} \mathrm{S}-73^{\circ} 00^{\prime}$ W)

Parque Nacional Monte León (= Monte León)

Parque Nacional Perito Moreno ( $47^{\circ} 57^{\prime}$ S - $72^{\circ} 15^{\prime}$ W)

Paso Ibáñez (ca. $50^{\circ} 10^{\prime} \mathrm{S}-70^{\circ} 00^{\prime} \mathrm{W}$ )

Perito Moreno ( $\left.46^{\circ} 38^{\prime} \mathrm{S}-70^{\circ} 54^{\prime} \mathrm{W}\right)$

Puerto Bandera (ca. $50^{\circ} 18^{\prime} \mathrm{S}-72^{\circ} 48^{\prime} \mathrm{W}$ )

Puerto Coig $\left(50^{\circ} 57^{\prime} \mathrm{S}-69^{\circ} 12^{\prime} \mathrm{W}\right)$

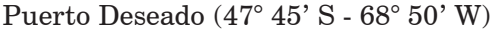

Puerto San Julián (ca. $49^{\circ} 18^{\prime} \mathrm{S}-67^{\circ} 43^{\prime} \mathrm{W}$ )

Puerto Santa Cruz ( $\left(50^{\circ} 01^{\prime} \mathrm{S}-68^{\circ} 31^{\prime} \mathrm{W}\right)$

Punta Bandera (50 $\left.18^{\prime} \mathrm{S}-72^{\circ} 48^{\prime} \mathrm{W}\right)$

Punta Loyola (ca. $51^{\circ} 38^{\prime} \mathrm{S}-69^{\circ} 05^{\prime} \mathrm{W}$ )

Punta Maqueda (46. $\left.02^{\prime} \mathrm{S}-67^{\circ} 35^{\prime} \mathrm{W}\right)$

Punta Quilla (ca. 50 $01^{\circ}$ ' S - $68^{\circ} 31^{\prime} \mathrm{W}$ )

Reserva de Vida Silvestre Los Escarchados (ca. 50 $\left.24^{\prime} \mathrm{S}-71^{\circ} 33^{\prime} \mathrm{W}\right)$

Ría Coig ( $\left.50^{\circ} 57^{\prime} \mathrm{S}-69^{\circ} 10^{\prime} \mathrm{W}\right)$

Ría Santa Cruz (50 $01^{\circ}$ S - $\left.68^{\circ} 31^{\prime} \mathrm{W}\right)$

Río Belgrano $\left(48^{\circ} 15^{\prime} \mathrm{S}-71^{\circ} 14^{\prime} \mathrm{W}\right)$

Río Blanco $\left(49^{\circ} 17^{\prime} \mathrm{S}-72^{\circ} 57^{\prime} \mathrm{W}\right)$

Río Bote $\left(50^{\circ} 16^{\prime} \mathrm{S}-71^{\circ} 44^{\prime} \mathrm{W}\right)$

Río Chico $\left(49^{\circ} 56^{\prime} \mathrm{S}-68^{\circ} 32^{\prime} \mathrm{W}\right)$

Río Coig (= Río Coyle) $\left(50^{\circ} 58^{\prime} \mathrm{S}-69^{\circ} 11^{\prime} \mathrm{W}\right)$

Río de las Vueltas $\left(49^{\circ} 30^{\prime} \mathrm{S}-72^{\circ} 50^{\prime} \mathrm{W}\right)$
Río Deseado ( $\left.46^{\circ} 34^{\prime} \mathrm{S}-70^{\circ} 31^{\prime} \mathrm{W}\right)$

Río Ecker ( $\left.47^{\circ} 04^{\prime} \mathrm{S}-70^{\circ} 45^{\prime} \mathrm{W}\right)$

Río Gallegos (51 $36^{\circ} \mathrm{S}-68^{\circ} 59^{\prime} \mathrm{W}$ )

Río Guanaco $\left(49^{\circ} 47^{\prime} \mathrm{S}-72^{\circ} 09^{\prime} \mathrm{W}\right)$

Río La Leona (ca. $50^{\circ} 13^{\prime} \mathrm{S}-72^{\circ} 25^{\prime} \mathrm{W}$ )

Río Mitre $\left(50^{\circ} 26^{\prime} \mathrm{S}-72^{\circ} 44^{\prime} \mathrm{W}\right)$

Río Pinturas $\left(46^{\circ} 35^{\prime} \mathrm{S}-70^{\circ} 18^{\prime} \mathrm{W}\right)$

Río Santa Cruz (500 $\left.08^{\prime} \mathrm{S}-68^{\circ} 20^{\prime} \mathrm{W}\right)$

Río Turbio ( $51^{\circ} 32^{\prime} \mathrm{S}-72^{\circ} 18^{\prime} \mathrm{W}$ )

San Julián $\left(49^{\circ} 18^{\prime}\right.$ S - $67^{\circ} 43^{\prime}$ W)

Santa Cruz (=Puerto Santa Cruz) (50 01' S - 68 $31^{\circ}$ W)

Sección Centro - Parque Nacional Los Glaciares (ca. $49^{\circ} 50^{\prime} \mathrm{S}-73^{\circ} 00^{\prime} \mathrm{W}$ )

Sierra Baguales (50 $49^{\prime} \mathrm{S}-72^{\circ} 09^{\prime} \mathrm{W}$ )

Tres Cerros ( $48^{\circ} 12^{\prime} \mathrm{S}-67^{\circ} 47^{\prime} \mathrm{W}$ )

Ventisquero Onelli (ca. $50^{\circ} 20^{\prime} \mathrm{S}-73^{\circ} 15^{\prime} \mathrm{W}$ )

\section{BIBLIOGRAPHY}

Administración de Parques Nacionales. 1980-90. Listado de observaciones de Guardaparques del Parque Nacional Los Glaciares, Delegación Regional Patagonia, (inédito).

Albrieu, C., S. Imberti \& S. Ferrari. 2004. Las aves del estuario del Río Gallegos, una guía de identificación. Universidad Nacional de la Patagonia Austral, Río Gallegos, 204 pp.

Babarskas, M. \& J.C. Chebez. 1999. Notas breves sobre aves de la Argentina y países vecinos. Nuestras Aves, 39: 12-14.

Burmeister, C.V. 1890. Expedición a Patagonia. Anal. Mus. Nac. Buenos Aires, 3: 253-321.

Cadierno, S.A. \& C.D. Amorós. 1999. Cauquén Colorado (Chloephaga rubidiceps) y Cabecitanegra Austral (Carduelis barbata) en la Estancia Cóndor, provincia de Santa Cruz, Argentina. Nuestras Aves, $\mathrm{N}^{\circ} 40: 16$.

Chebez, J.C., C. Bertonatti, A. Johnson, S. Heinonen Fortabat \& G. Gil. 1988. Notas sobre la distribución de algunas aves santacruceñas. APRONA, 8: 1427.

Chebez, J.C., N.R. Rey, M. Babarskas \& A.G. Di Giacomo.1998. Las aves de los parques nacionales de la Argentina. Monografía LOLA, 12: 126 pp.

Chesser, R.T. \& M.A. Marín. 1994. Seasonal distribution and natural history of the Patagonian Tyrant (Colorhamphus parvirostris). Wilson Bull. 106 (4): 649-667.

Coconier, E. 2005. Aves Acuáticas en la Argentina. Reporte Final, Aves Argentinas-Wetlands International, 137 pp. www.avesargentinas.org.ar

Contreras, J.R. 1980. Furnariidae Argentinos. I. Nuevos datos sobre Tripophaga modesta navasi y algunas consideraciones sobre Tripophaga modesta en la Argentina. Historia Natural, 1(9): 49-68.

Dabbene, R. 1919. Las especies y subespecies argentinas de los géneros Geositta Swainson y Cinclodes Gray. Anal. Mus. Nac. Hist. Nat. Buenos Aires, 30: 113196.

- 1933. Notas sobre las especies argentinas del género Phrygilus. Anal. Soc. Cient. Argent. 115: 169-193, 297-324. 
Daciuk, J. 1977. Notas faunísticas y bioecológicas de península Valdés y Patagonia. VI. Observaciones sobre areas de nidificación de la avifauna del litoral marítimo paragónico (provincias de Chubut y Santa Cruz, Rep. Argentina). Hornero, 11(5): 361-376.

Darrieu, C.A., A.R. Camperi \& S. Imberti. 2008. Avifauna (Non Passeriformes) of Santa Cruz province, Patagonia (Argentina): annotated list of species. Rev. Mus. Argentino Cienc. Nat., n.s., 10(1): 111-145.

De la Peña, M.R. 1985. Notas nidobiológicas sobre aves argentinas. Familia: Tyrannidae. Nuestras Aves, (8): 13-14.

- 1986. Notas nidobiológicas sobre aves argentinas. Familia: Tyrannidae. Nuestras Aves, (9): 14-15.

- 1987. Nidos y huevos de aves argentinas. Ed. del autor, $260 \mathrm{pp}$.

- 2005. Reproducción de las aves argentinas (con descripción de pichones). L.O.L.A. 845 pp.

De Lucca, E.R. \& M.D. Saggese. 1992. Aves del departamento Deseado, Santa Cruz. Hornero, 13(3): 259-260.

De Lucca, E.R., M. Saggese \& M. Ióppolo. 1993. Proyecto Aguila Escudada. Campaña 1987-1988. Primera Reunión de Ornitología de la Cuenca del Plata. Resúmenes: 14.

Del Hoyo, J., A. Elliott \& D. Christie. 2003. Handbook of the birds of the world. Vol 8. Broadbill to Tapaculos. Lynx Edicions. Barcelona. 845 pp.

- 2004. Handbook of the birds of the world. Vol 9. Cotingas to Pipits and Wagtails. Lynx Edicions. Barcelona 863 pp.

- 2005. Handbook of the birds of the world. Vol 10. Cuckoo-shrikes to Thrushes. Lynx Edicions. Barcelona. 895 pp.

Del Hoyo et al., Handbook of the birds of the world. Vol. 11. Old World Flycatchers to Old World Warblers. Lynx Edicions. Barcelona. 798 pp.

Di Giácomo, A.S., V. De Francesco \& E.G. Coconier. 2005. Areas importantes para la conservación de las aves en la Argentina. Aves Argentinas. CD.

Earnshaw, A. \& N. Earnshaw. 2000. Lista de aves avistadas en un viaje a la Patagonia- enero 2000. www.geocities.com/viajesnat/patagonia2000/p2000li.htm

Erize, F. 1983. Observaciones sobre el Macá Tobiano. Hornero, $N^{\circ}$ extraordinario, 256-268.

Esteban, J.G. 1951. "Furnariinae" de la República Argentina. Acta Zool. Lilloana, 12: 377-441.

Fraga, R. \& S. Narosky. 1985. Nidificación de las aves argentinas (Formicariidae a Cinclidae). Asociación Ornitológica del Plata, 96 pp.

Gould, J. \& C. Darwin. 1838-1841. Birds. En C. Darwin (ed.), Zoology of H.M.S. Beagle. 1832 to 1836, 1: 164.

Hellmayr, C.E. 1925. Catalogue of birds of the Americas and the adjacent islands. Field Mus. Nat. Hist., (Furnariidae-Dendrocolaptidae): 13(4): 1-390.

- 1938. Catalogue of birds of the Americas and the adjacent islands. Field Mus. Nat. Hist., ( PloceidaeCatamblyrhynchidae-Fringillidae): 13(11): 1-662.

Humphrey, P.S. \& D. Bridge. 1970. Apuntes sobre distribución de aves en la Tierra del Fuego y la Patagonia argentina. Revista del Museo Argentino de Ciencias Naturales Bernardino Rivadavia, N.S., Zoología, 10(17): 251-265.

Humphrey, P.S., D. Siegel-Causey \& P.C. Rasmusenn. 1985. Report on Ornithological Research conducted in the provinces of Chubut and Santa Cruz, Argentina, December 1984 through February 1985. Museum of Natural History University of Kansas, 38 pp.

Iglesias, G.J. \& A.A. Pérez. 1998. Región 4: Patagonia. En: P. Canevari, D.E. Blanco, E.H. Bucher, G. Castro \& I. Davidson (eds.), Los Humedales de la Argentina: Clasificación, Situación Actual, Conservación y Legislación. Wetlands International Publ. 46, Buenos Aires, Argentina. 208 pp.

Imberti, S. 2003. Notes on the distribution and natural history of some birds in Santa Cruz and Tierra del Fuego provinces, Patagonia, Argentina. Cotinga, 19: 15-24.

- 2005. Aves de Los Glaciares: Inventario Ornitológico del Parque Nacional Los Glaciares, Santa Cruz, Patagonia, Argentina. Aves Argentinas \& APN. 79 pp.

Lista de aves de la Reserva Provincial Cabo Virgenes. (Informe inédito/Unpublished report a), 7 pp.

- Lista de aves zona Estancia La Angostura. (Informe inédito/Unpublished report b), 3 pp.

Imberti, S. \& C. Albrieu. 2001. Lista de las aves de Laguna Nimez. Universidad Nacional de la Patagonia Austral. 14 pp.

Lange, C. 1981. Una temporada de observaciones sobre Podiceps gallardoi (Aves: Podicipediformes). Ecología y etología. Neotrópica, 27(77): 39-56.

Maclean, G. 1969. The nest and eggs of the Chocolate Tyrant Neoxolmis rufiventris (Vieillot). Auk, 86: 144-145.

Mazar Barnett, J. \& M. Pearman. 2001. Lista comentada de las aves argentinas. Lynx Edicions, 164 pp.

Mazar Barnett, J., M. della Seta, S. Imberti \& G. Pugnali. 1998. Notes on the rediscovery of the Austral Rail Rallus antarcticus in Santa Cruz, Argentina. Cotinga, 10: 96-101.

Narosky, S., R. Fraga \& M. de la Peña. 1983. Nidificación de las aves argentinas (Dendrocolaptidae y Furnariidae). Asoc. Ornit. del Plata, 98 pp.

Navas, J.R. 1965. El área geográfica de Phytotoma rara (Aves, Phytotomidae). Neotrópica, 2(34): 38-40. 1971. Estudio sobre la avifauna andinopatagónica. I. Géneros Upucerthia, Ochetorhynchus y Eremobius (Furnariidae). Revista del Museo Argentino de Ciencias Naturales Bernardino Rivadavia, N.S., Zoología, 7(3): 269-304.

Navas, J.R. \& N.A. Bó. 1987. Notas sobre Furnariidae argentinos (Aves, Passeriformes). Revista del Museo Argentino de Ciencias Naturales Bernardino Rivadavia, N.S., Zoología, 14(4): 55-86.

1991. La distribución geográfica de las razas australes de Anairetes parulus en la Argentina (Aves, Tyrannidae). Neotrópica, 37(98): 145-149. 1994. Apuntes sobre distribución y cría de algunas especies de los géneros Agriornis, Xolmis, Neoxolmis y Muscisaxicola (Aves, Tyrannidae). Revista del Museo Argentino de Ciencias Naturales Bernardino Rivadavia, 16(7): 81-96. 
Nores, M. y D. Yzurieta. 1995. Nuevas localidades para aves argentinas. Parte VIII. Hornero, 14(1 y 2): 72-73.

Olrog, C.C. 1948. Observaciones sobre la avifauna de Tierra del Fuego y Chile. Acta Zoológica Lilloana, 5: 437-531.

Oustalet, E. 1891. Misión Scientifique du Cap Horn, 1882-83. VI. Zool., Oiseaux, 341 pp.

Paynter, R.A. Jr. 1995. Ornithological gazetteer of Argentina. 2nd edition. Cambridge, Massachusetts. $1043 \mathrm{pp}$.

Pergolani de Costa, M.J.I. 1946. Los Agriornis argentinos. Revisión del género Agriornis Gould, Familia Tyrannidae. Ministerio de Agricultura, Buenos Aires, ser. A, (13): 16.

- 1949. Los Xolmis argentinos. Revisión del género Xolmis Boie. Familia Tyrannidae, Orden Passeriformes. Ministerio de Agricultura, Buenos Aires, ser. A, (47): 36.

- 1975. Los Muscisaxicola argentinos. Revisión del género Muscisaxicola Lafresnaye y d'Orbigny, Familia Tyrannidae, Orden Passeriformes. Hornero, 11(4): 242-254.

Piloni, G. 2002 (2004). Revisión sistemática de las subespecies de Zonotrichia capensis (P. L. S. Müller) (Aves: Emberizinae) y su distribución geográfica en la República Argentina. Physis, Secc. C, 60(138139): 1-23.

Pozzi, A. 1923. La perdiz de Santa Cruz (Patagonia) Tinamotis ingoufi Oust. Hornero, 3(2): 180-184.

Radboone, S. 1935. Notas sobre algunas aves del lago San Martín (Santa Cruz). Hornero, 6(1): 99-101.

Ridgely, R.S. \& G. Tudor. 1994. The birds of South America. Vol. 1. The Oscines Passerines. Oxford University Press. 516 pp.

Saggese, M.D. \& R. De Lucca. 2001. Biología reproductiva del Aguila Mora (Geranoaetus melanoleucus) en la Patagonia sur, Argentina. Hornero, 16(2): 77-84.

Salvadori, T. 1900. Contribuzione all' avifauna dell' America Australe (Patagonia, Terra del Fuoco, Isola
Degli Stati, Isole Falkland). Annali del Museo Civico di Storia Naturale di Genova, Ser.2 (20): 609-634.

Sclater, P.L. 1874. On the species of the genus Synallaxis of the family Dendrocolaptidae. Proceedings of the Zoological Society of London, 1874: 228.

- 1888. Catalogue of the Passeriformes in the Collection of the British Museum. Oligomyoda, Vol. 14 495 pp. London.

- 1890. Catalogue of the Passeriformes or perching birds in the Collection of the British Museum. Tracheophonae. Vol. 15. London. 484 pp.

Scott, W.E.D. 1900a. Notes on three new species of Tyrannidae from Patagonia. Bulletin of British Ornithological Club, (10): 54-55.

- 1900b. New or undetected species of birds from South America. Bulletin of British Ornithological Club, (10): 62-64.

Stone, W. 1928. Reports of the Princeton University Expedition to Patagonia, 1896-1899. Ornithology, 2(5): 719-857.

Wetmore, A. 1926. Report on a collection of birds made by J. R. Pemberton in Patagonia. University of California Publication in Zoology, 24: 395-474.

Zapata, A.R.P. 1967. Observaciones sobre aves de Puerto Deseado provincia de Santa Cruz. Hornero, 10(4): 351-378.

- 1969. Aves observadas en el Golfo San Jorge, Provincias de Chubut y Santa Cruz, Argentina. Zoología Platense, 1(5): 21-27.

Zapata, A.R.P. \& H.S. Martínez. 1972. Algunas aves no citadas y otras pocos frecuentes para el sur de la provincia de Buenos Aires. Acta Zoológica Lilloana, 29: 181-199.

Zotta, A.R. 1937. Una nueva subespecie de pecho colorado Pezites militaris catamarcanus, subsp. nov. Hornero, 6(3): 449-454.

- 1940. Notas ornitológicas. Distribución de Melanodera xanthogramma barrosi (Chapman). Hornero, 7(3): 359-365. 